ARTICLE

https://doi.org/10.1038/s41467-020-18054-y

\title{
TET1 is a beige adipocyte-selective epigenetic suppressor of thermogenesis
}

Sneha Damal Villivalam¹, Dongjoo You', Jinse Kim¹, Hee Woong Lim (1D 2, Han Xiao, Pete-James H. Zushin', Yasuo Oguri ${ }^{3}$, Pouya $\operatorname{Amin}^{1} \&$ Sona Kang (10 ${ }^{1 凶}$

It has been suggested that beige fat thermogenesis is tightly controlled by epigenetic regulators that sense environmental cues such as temperature. Here, we report that subcutaneous adipose expression of the DNA demethylase TET1 is suppressed by cold and other stimulators of beige adipocyte thermogenesis. TET1 acts as an autonomous repressor of key thermogenic genes, including Ucp1 and Ppargc1a, in beige adipocytes. Adipose-selective Tet1 knockout mice generated by using Fabp4-Cre improves cold tolerance and increases energy expenditure and protects against diet-induced obesity and insulin resistance. Moreover, the suppressive role of TET1 in the thermogenic gene regulation of beige adipocytes is largely DNA demethylase-independent. Rather, TET1 coordinates with HDAC1 to mediate the epigenetic changes to suppress thermogenic gene transcription. Taken together, TET1 is a potent beige-selective epigenetic breaker of the thermogenic gene program. Our findings may lead to a therapeutic strategy to increase energy expenditure in obesity and related metabolic disorders.

\footnotetext{
${ }^{1}$ Nutritional Sciences and Toxicology Department, University of California Berkeley, Berkeley, CA 94720, USA. ${ }^{2}$ Division of Biomedical Informatics, Cincinnati Children's Hospital Medical Center Department of Pediatrics \& Biomedical Informatics, University of Cincinnati, 3333 Burnet Ave. MLC 7024, Cincinnati, OH 45229, USA. ${ }^{3}$ UCSF Diabetes Center, Eli and Edythe Broad Center of Regeneration Medicine and Stem Cell Research, Department of Cell and Tissue Biology,

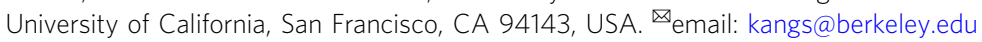


M ammals have at least two types of thermogenic adipocytes, brown and beige, that play a central role in regulating energy homeostasis ${ }^{1,2}$. In rodents, classical brown adipose tissue (BAT) exists in discrete anatomical depots, such as the interscapular regions, while beige adipocytes sporadically arise within white adipose tissue (WAT) $)^{1,2}$. These two thermogenic adipocyte subtypes are similar in multiple respects: both contain multilocular lipid droplets, have a high mitochondrial content, and express key thermogenic genes such as Ucp1, Ppargcla, and Cidea ${ }^{1,2}$. At the same time, beige and brown adipocytes have several distinct characteristics that distinguish them as two different cell types. For instance, their developmental origins are different. While classical brown adipocytes derive from Myf5-positive precursors during embryonic development, beige adipocytes postnatally develop in the WAT depots of adults and derive from multiple origins depending on the $\operatorname{depot}^{3-7}$. In addition, molecular profiling studies highlight significant differences between the gene signatures of brown vs. beige adipocytes ${ }^{8,9}$. Lastly, the plasticity of thermogenic activity remarkably differs between these two cell types. Brown adipocytes are constitutively active and express high levels of UCP1 and other thermogenic genes. Their thermogenic activity can be further increased to a moderate degree upon stimulation. On the other hand, beige adipocytes express very low levels of thermogenic genes but robustly induce their expression in response to external stimuli, thus displaying a greater degree of thermogenic plasticity ${ }^{1,2}$.

Beige fat formation, also called "browning" or "beiging", is induced by various environmental cues, including cold exposure, exercise, and PPAR $\gamma$ agonist ${ }^{1,2}$, and it results in the production of heat by burning stored fat. Conversely, beige adipocytes also undergo "whitening", returning them to a white adipocyte-like phenotype, in response to thermoneutrality ${ }^{10}$, impaired $\beta$ adrenergic signaling ${ }^{11}$, triglyceride hydrolase deficiency ${ }^{12}$, and other cues ${ }^{13}$. In mice, beige fat contains one tenth the amount of UCP1, a key thermogenic protein, than brown fat ${ }^{14}$ however, the total amount of beige fat can be greater than brown fat and can have a bigger impact on energy homeostasis, as it can be recruited en masse in many white depots ${ }^{14}$. Notably, recent studies have identified additional mechanisms through which beige fat modulates whole-body metabolism such as creatine cycling ${ }^{15}$ and Serca2b-mediated calcium cycling ${ }^{16}$. In humans, although still debatable, a substantial number of studies suggest that thermogenic adipocytes are recruited upon cold acclimatization ${ }^{17,18}$ leading to increased energy expenditure and a beneficial impact on glucose metabolism ${ }^{18,19}$. Due to this remarkable plasticity and the relevance to human obesity, beige adipocytes are an attractive therapeutic target for obesity and related metabolic diseases.

The browning and whitening of beige and brown fat involves dramatic changes in morphology, transcription, and chromatin landscape ${ }^{10,20-25}$. Therefore, epigenetic regulators are likely to play a key role in this process. The ten-eleven translocation (TET) proteins (TET1, 2, and 3) are the enzymes that oxidize methylated cytosine. In addition to participating in the initial step of DNA demethylation, TETs play versatile roles in transcription regulation $^{26}$ by acting as both transcriptional co-activators and corepressors ${ }^{26}$. Notably, the transcriptional regulation activity of TET proteins can be dependent or independent of their demethylase activity through interacting with other transcriptional regulators and chromatin modifiers ${ }^{27-31}$.

Here, we report that TET1 expression in subcutaneous adipose tissue shows reciprocal regulation with UCP1 in response to ambient temperature changes and cAMP signaling. We demonstrate that Tet1 loss-of-function leads to cell-autonomous increases in cAMP-induced expression of thermogenic genes, including $U c p 1$, and increases in mitochondrial respiration in beige adipocytes. Consistent with this, conditional deletion of
Tet1 in adipose tissue increases browning of subcutaneous fat, leading to reduced adiposity and improved cold tolerance, glucose tolerance, and insulin sensitivity. Moreover, the knockout mice are protected from diet-induced obesity and metabolic impairment. Mechanistically, we identify that TET1 collaborates with HDAC1 to mediate the epigenetic changes that suppress thermogenic gene transcription in a DNA demethylase-independent manner. Together, our results suggest that TET1 is a potent epigenetic sensor of ambient temperature and modulates the temperature-mediated browning of beige adipocytes.

\section{Results}

Adipose TET1 levels in response to temperature and cAMP First, we compared the expression of Tets across tissues and noted that all three Tets are moderately expressed in inguinal and epididymal white adipose tissues (iWAT and eWAT) and less expressed in BAT (Fig. 1a-d). To identify a potential role for the DNA methylation machinery in the temperature-induced plasticity of beige and brown adipocytes, we examined how TET expression in adipose tissue is affected by changes in ambient temperature. Inguinal white adipose tissue (iWAT), epididymal WAT (eWAT), interscapular brown fat tissue (BAT) were taken from wild-type $\mathrm{C} 57 \mathrm{BL} / 6 \mathrm{~J}$ mice that were housed at RT $\left(23^{\circ} \mathrm{C}\right)$ before being subjected to cold $\left(4^{\circ} \mathrm{C}\right)$ or thermoneutral (TN, $30^{\circ}$ C) temperatures for $24 \mathrm{~h}$. As expected, $U c p 1$ levels overall went up in all three depots when mice were housed for $24 \mathrm{~h}$ at cold $\left(4^{\circ} \mathrm{C}\right)$ and down when housed at thermoneutrality $\left(\mathrm{TN}, 30^{\circ} \mathrm{C}\right.$ ) (Fig. 1e, Supplementary Fig. 1a, e). Importantly, the expression of Tet1 was most dramatically regulated by the changes in ambient temperature, especially in iWAT (Fig. 1f). Heat increased the mRNA expression of Tet 1 by $\sim 20$ fold whereas cold reduced it by $\sim 60 \%$ (Fig. 1f). The temperature-sensitive regulation of TET1 in iWAT was also confirmed by western blotting (Fig. 1f). A similar expression pattern was observed in BAT and eWAT albeit smaller in magnitude (Supplementary Fig. 1b, f).

Similar to Tet1, the expression of Tet2 was also changed but to a lesser degree in iWAT (Fig. 1g, Supplementary Fig. 1c, g). In contrast, Tet3 levels did not show consistent changes between depots; they had a $\sim 3$ fold increase in iWAT (Fig. 1h), a decrease in BAT (Supplementary Fig. 1d), and no change in eWAT (Supplementary Fig. 1h) under cold conditions. The anticorrelation between Tet1 levels with Ucp1 in vivo prompted us to determine their expression levels in three different shades of adipocyte cell lines: 3T3-L1 cells (considered "white") and immortalized "beige" and "brown" adipocytes. As expected, UCP1 protein expression was not detectable in mature 3T3-L1 adipocytes, had intermediate expression in mature beige adipocytes, and high expression in mature brown adipocytes (Fig. 11). Interestingly, TET1 expression was increased during white adipogenesis and reduced during beige and brown adipogenesis, displaying an anti-correlation with UCP1 levels (Fig. 1i, l). On the other hand, both the expression of TET2 and TET3 was overall higher in all three types of mature adipocytes compared to that of preadipocytes (Fig. 1j-1). As we noted that Tet1 expression was relatively higher in iWAT compared to BAT (Fig. 1a, d), we looked into how the regulation of Tet 1 expression is regulated in response to thermogenic stimulators (cAMP-inducing forskolin and norepinephrine) in in vitro-differentiated primary inguinal adipocytes. Consistent with in vivo, Tet1 mRNA expression was reciprocally regulated with $U c p 1$ expression in response to the thermogenic stimulators (Fig. 1m, n, Supplementary Fig. 1i, j). Together, our expression data suggested that TET1 and TET2 have a functional role in the regulation of thermogenesis in beige fat, thus we sought to test the effects of their downregulation in beige adipocyte thermogenesis. 
a

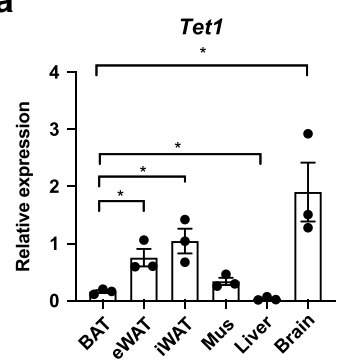

e

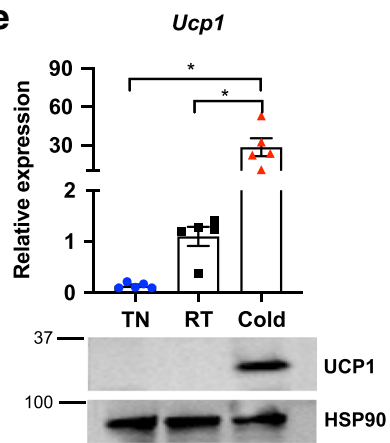

b

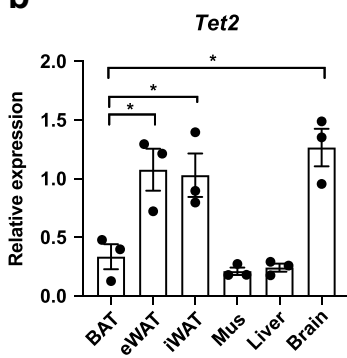

f

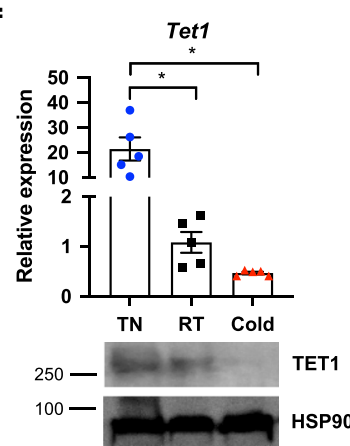

C

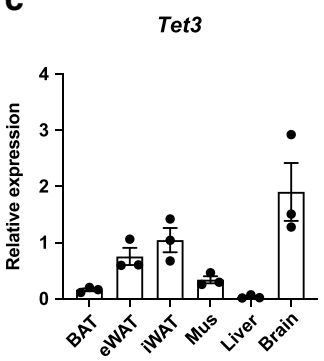

g

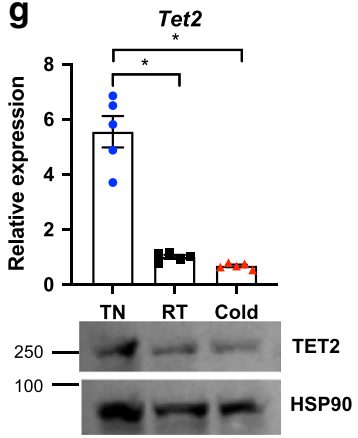

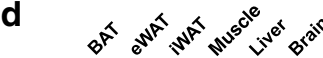
$250-$ $250-\square=-7$ TET2 $250-2-2-$ TET3 100 - Hoto HSP90

h

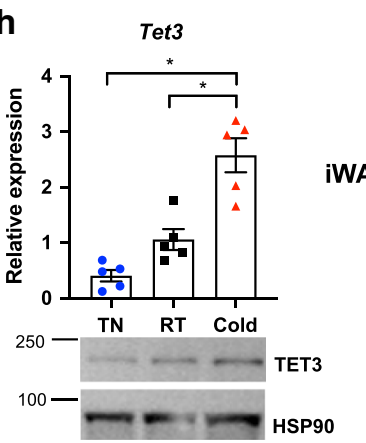

i $\quad$ jet1

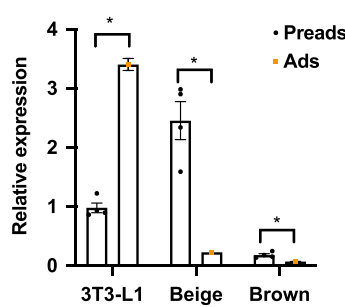

j Tet2

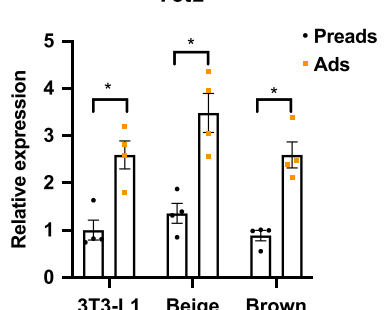

k

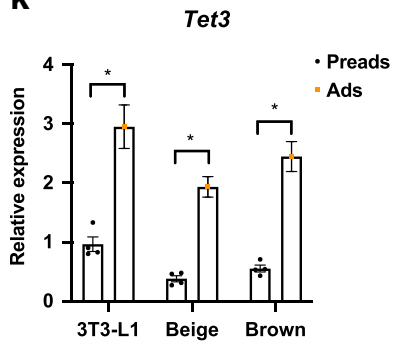

I

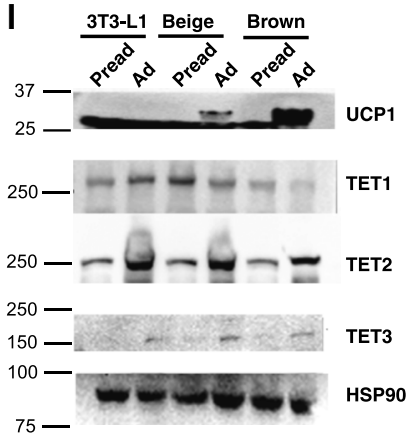

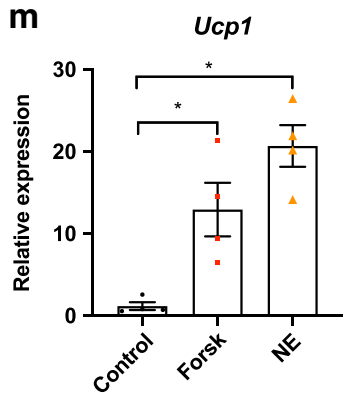

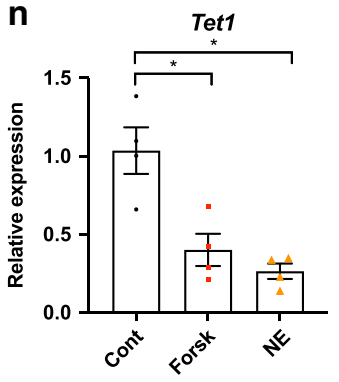

Primary beige adipocytes

Fig. 1 Subcutaneous adipose Tet1 expression is regulated by ambient temperature and cAMP signaling. a-d Various tissues from wild-type C57BL/6 $J$ mice housed at room temperature (RT) were collected to measure Tets mRNA (a-c) and protein expression $\mathbf{d}(\mathbf{a}-\mathbf{c}) ; n=3$ per group. Data are expressed as means \pm SEM. *denotes $p<0.05$, determined by two-tailed Student's $t$ test and one-way ANOVA). e-h Tet and Ucp1 mRNA and protein expression in iWAT from wild-type male C57BL/6J mice housed at room temperature (RT) and exposed to cold or thermoneutrality (TN) for $24 \mathrm{~h}(n=5 \mathrm{per}$ group. Data are expressed as means \pm SEM. *denotes $p<0.05$, determined by two-tailed Student's $t$ test and one-way ANOVA followed by Bonferroni post-hoc testing). $\mathbf{i}-\mathbf{I}$ Tets mRNA and protein expression were measured in 3T3-L1 and immortalized beige and brown preadipocytes at confluence and after differentiation (i-k; $n=4$ per group. Data are expressed as means \pm SEM. *denotes $p<0.05$, determined by two-tailed Student's $t$ test). $\mathbf{m}, \mathbf{n} U c p 1$ and Tet 1 mRNA expression with and without $1 \mu \mathrm{M}$ forskolin (Forsk) or $1 \mu \mathrm{M}$ norepinephrine (NE) stimulation for $3 \mathrm{~h}$ in mature primary beige adipocytes ( $n=4$ per group, Data are expressed as means \pm SEM. ${ }^{*}$ denotes $p<0.05$, determined by two-tailed Student's $t$ test and one-way ANOVA followed by Bonferroni post-hoc testing). Source data are provided as a source data file.

TET1 suppresses the thermogenic activation of beige adipocytes. To test the cell-autonomous role of TETs in the regulation of thermogenic genes, we performed gain- and loss-of-function studies of individual TETs using an immortalized beige cell line. Since TETs are pro-adipogenic ${ }^{32}$ we conducted the studies in fully mature beige adipocytes using short hairpin RNAs (shRNAs)
(Supplementary Fig. 2a-c) to avoid any effect on differentiation. Remarkably, knockdown of Tet 1 , but not Tet 2 or Tet 3 , enhanced the forskolin-stimulated expression of $U c p 1$ and some of key thermogenic markers including Ppargcla, Cidea, and Elovl3 (Fig. $2 \mathrm{a}-\mathrm{d}$ ). Notably, there was no change in the expression levels of general adipocyte markers such as Pparg and Fabp4 (Fig. 2e, f). 
a

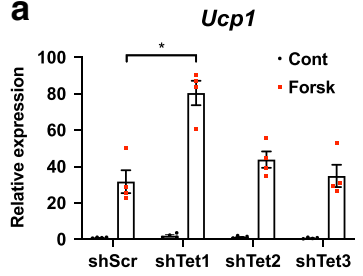

e

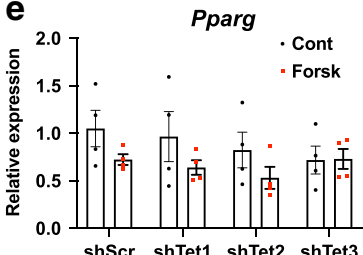

b

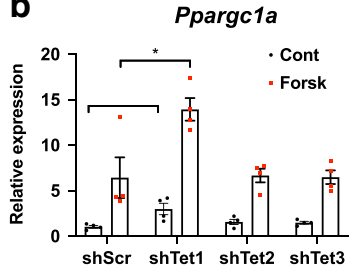

f

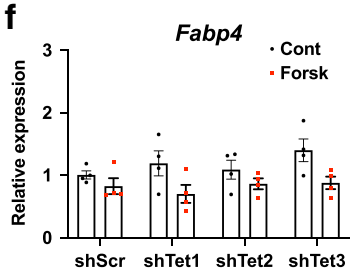

c

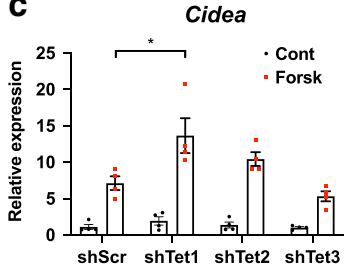

d

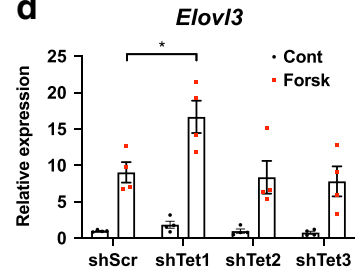

g

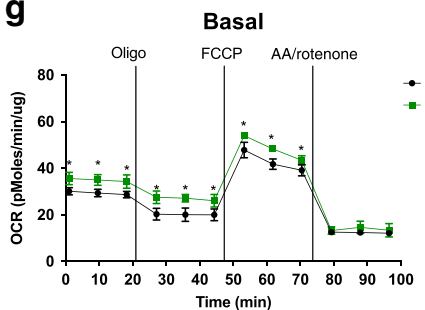

h

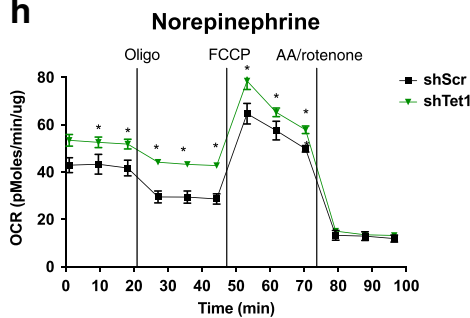

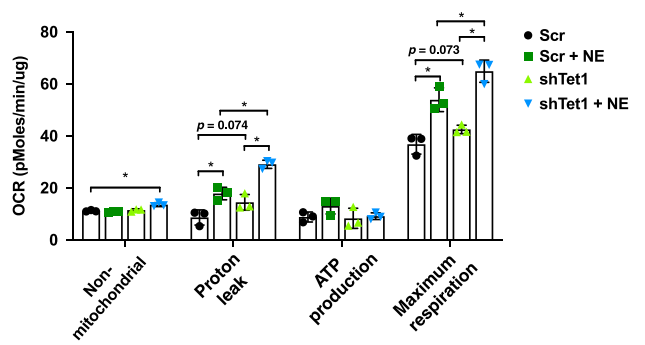

Fig. 2 Tet1 loss-of-function in vitro increases thermogenesis in beige adipocytes. a-f Differentiated beige adipocytes were transduced with hairpins against Tet1, 2, and 3 and scrambled control shRNA (shScr). The basal- and forskolin-stimulated levels of key adipocyte thermogenic gene transcripts were measured by qPCR ( $n=4$ per group. Data are expressed as means \pm SEM. *denotes $p<0.05$, determined by two-tailed Student's $t$ test and two-way ANOVA followed by Bonferroni post-hoc testing). g-i Basal and norepinephrine (NE)-stimulated mitochondrial respiration under various drug treatments was measured in Tet 1 knockdown and scramble beige adipocytes ( $n=3$ per group. Data are expressed as means \pm SEM. *denotes $p<0.05$, determined by two-tailed Student's $t$ test and one-way ANOVA). (Oligo; Oligomycin, AA; antimycin A). i Shown are the various components of oxygen consumption rates with and without NE stimulation from $(\mathbf{g}, \mathbf{h})$. ( $n=3$ per group. Data are expressed as means \pm SEM. *denotes $p<0.05$, determined by two-tailed Student's $t$ test and two-way ANOVA followed by Bonferroni post-hoc testing). Source data are provided as a source data file.

Tet1 knockdown in beige adipocytes increased the rate of mitochondrial respiration in the presence and absence of norepinephrine (Fig. 2g-i). Conversely, lentiviral overexpression of Tet1 in mature beige adipocytes (Supplementary Fig. 2d, e) suppressed the expression of thermogenic genes (Fig. 3a-d) without altering the expression of general adipocyte markers (Pparg and Fabp4) (Fig. 3e, f) and suppressed mitochondrial respiration in the presence and the absence of norepinephrine (Fig. 3g-i). Together, our results suggest that TET1 suppresses adipocyte thermogenesis in a cell-autonomous manner in beige adipocytes.

Tet1 KO mice display increased cold tolerance and energy expenditure. To determine the in vivo role of Tet1 in the regulation of adipose plasticity and the thermogenic gene program, we initially generated adipose-selective Tet 1 knockout mice (AdipoQ-Tet1KO) using adiponectin-Cre mice ${ }^{33}$. However, these knockout mice had poor knockdown efficiency ( $10 \%)$ even when specifically assessing adipocytes (Supplementary Fig. 3a, b). Therefore, we used Fabp4-Cre ${ }^{34}$ to generate an adipose-selective knockout (Adi-Tet1KO) mouse, in which Tet1 mRNA levels were knocked down by more than $80 \%$ in the purified adipocyte fraction obtained from iWAT, eWAT, and BAT (Supplementary Fig. 3c, d). Since there is concern over the target specificity of Fabp4-Cre ${ }^{35}$, we thoroughly examined Tet1 knockdown in multiple other tissues. Other than $12 \%$ less expression in the brain, we did not find any significant changes in Tet1 expression in non-adipose tissues including the stromal-vascular fraction
(Supplementary Fig. 3c, d), which contains macrophages and precursors.

To examine whether TET1 is necessary for temperaturemediated adipose plasticity, we exposed Adi-Tet1KO and WT mice to different ambient temperatures. To assess their cold tolerance, we monitored the rectal temperature of individual mice placed into a $4{ }^{\circ} \mathrm{C}$ cold chamber. Adi-Tet $1 \mathrm{KO}$ mice maintained their core body temperature better than WT mice under cold conditions (Fig. 4a). Consistent with the increased tolerance to cold, the KO mice had a significant increase in oxygen consumption upon cold exposure and a trend towards increased oxygen consumption at RT and TN, as compared to controls (Fig. 4b, c). In addition, the serum release of glycerol was elevated in $\mathrm{KO}$ mice upon cold exposure (Fig. 4d), likely due to increased lipolysis to provide fuel for the increased adaptive thermogenesis. There were no changes in food intake, physical activity, or respiratory exchange ratio (RER) between genotypes (Supplementary Fig. $4 \mathrm{a}-\mathrm{c}$ ). We also noted that KO iWAT had more multilocular brown-like adipocytes after 7 days of cold exposure (Fig. 4e), whereas there was no marked difference between WT and KO BAT (Fig. 4e). Also notably, the increased rates of basal and norepinephrine-stimulated respiration was more pronounced in the KO iWAT than in the KO BAT (Fig. 4f, Supplementary Fig. 5a). This depot-biased effect was also observed at molecular levels; increased expression of UCP1 and PGC1a was found in iWAT but not in BAT (Fig. 4g, Supplementary Fig. 5b). This suggests that TET1 is acting selectively on beige adipocytes, which are present in iWAT. 
a
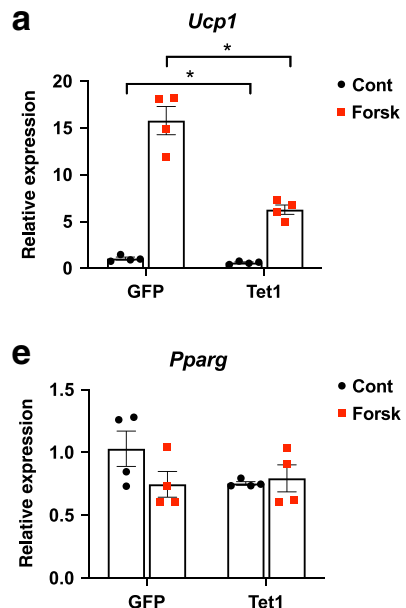

g

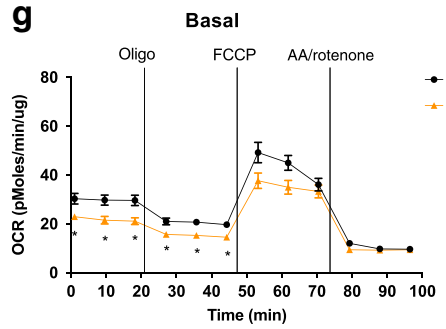

b

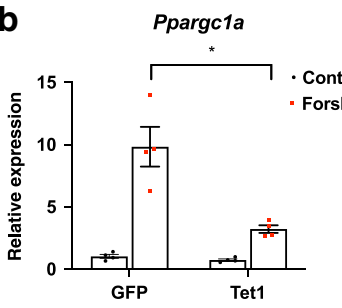

f

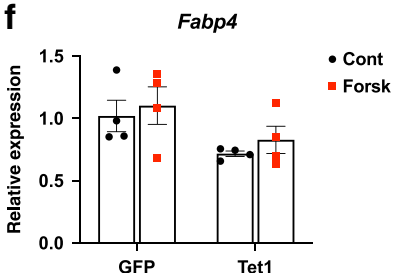

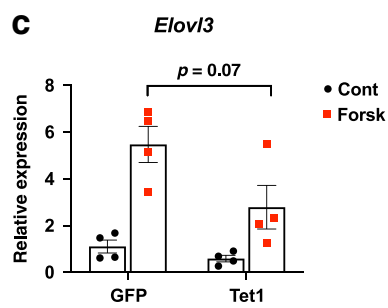

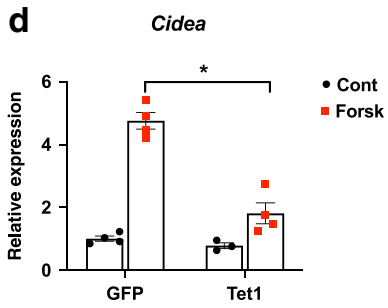

h
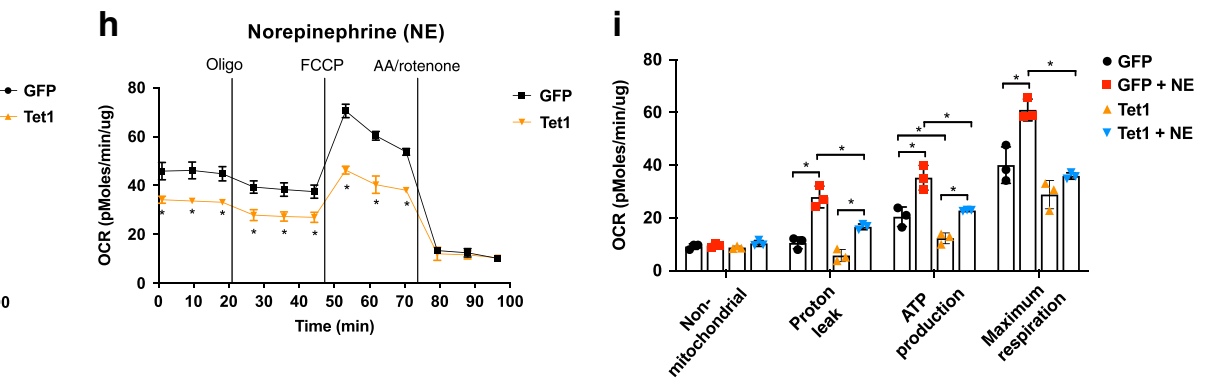

Fig. 3 Tet1 gain-of-function in vitro suppresses thermogenesis in beige adipocytes. a-f Differentiated beige adipocytes were transduced with lentiviral expression plasmids for Tet1 and GFP. The basal- and forskolin-stimulated levels of key adipocyte thermogenic gene transcripts were measured by qPCR $(n=4$ per group. Data are expressed as means \pm SEM. *denotes $p<0.05$, determined by two-tailed Student's $t$ test and two-way ANOVA followed by Bonferroni post-hoc testing). $\mathbf{g}$, h Basal and norepinephrine (NE)-stimulated mitochondrial respiration under various drug treatments was measured in Tet 1 overexpression and GFP beige adipocytes ( $n=3$ per group. Data are expressed as means \pm SEM. * denotes $p<0.05$, determined by two-tailed Student's $t$ test and one-way ANOVA). (Oligo; Oligomycin, AA; antimycin A). i Shown are the various components of oxygen consumption rates with and without NE stimulation from $\mathbf{g}, \mathbf{h}$. ( $n=3$ per group. Data are expressed as means \pm SEM. *denotes $p<0.05$, determined by two-tailed Student's $t$ test and two-way ANOVA followed by Bonferroni post-hoc testing). Source data are provided as a source data file.

Since Fapb4-Cre is also expressed in non-adipose tissues, we did a series of experiments to exclude the possibility of contributing factors from other cell types. It has been proposed that alternative M2 macrophage polarization can induce white adipose tissue browning ${ }^{36}$. Since Fabp4-Cre is active in macrophages, we tested whether there is a contribution from macrophages to the browning phenotype in the KO mice. First, we conducted FACS analysis, finding no major difference in M1 vs. M2 macrophage populations (Supplementary Fig. 6a). Second, we isolated primary macrophages from WT and KO mice and cocultured them with wild-type beige adipocytes using a transwell system (Supplementary Fig. 6b). We did not find any discernable changes in the expression of thermogenic genes between genotypes (Supplementary Fig. 6c).

PDGFRa-Cre is often used to target the adipocyte precursor population, and indeed, we were able to see $\sim 60 \%$ knockdown in the SVF population, defined by the absence of Lin markers (CD31, CD45, Ter119) (Supplementary Fig. 7a). Additionally, PDGFRa is expressed in selected cell types in a wide array of mesenchymal tissues, including the lung, heart, intestine, skin, and cranial facial mesenchyme as well as some adipocyte precursor cells ${ }^{37-39}$. We generated Tet1 $\mathrm{KO}$ mice using PDGFRa-Cre and found no remarkable differences in the thermogenic gene expression of iWAT and BAT in response to $\beta 3$-agonist or CL316,243 between genotypes (Supplementary Fig. 7b, c). Moreover, PDGFRa-Tet1 KO mice did not show any noticeable phenotypic changes in body weight (Supplementary Fig. 7d, h), body composition (Supplementary Fig. 7e, i), or oxygen consumption (Supplementary Fig. 7f, g) on both chow and HFD. In accordance with this, PDGFRa-Tet1 KO mice did not display major differences in insulin tolerance and glucose tolerance tests on chow and HFD (Supplementary Fig. 7j-1).

Last, we knocked down Tet1 expression selectively in inguinal adipose by using adeno-associated virus serotype 8 (AAV8)-Cre, whose expression is driven by the human adiponectin promoter ${ }^{40,41}$. To increase target specificity, we directly injected viruses into the inguinal fat pads of Tet $1 \mathrm{f} / \mathrm{f}$ mice. As a result, Tet 1 expression was $\sim 60 \%$ knocked down in iWAT but not in other tissues including eWAT and BAT (Supplementary Fig. 8a). To assess thermogenesis, AAV8-treated mice were treated with CL316,243, which resulted in increased signs of browning of iWAT at histological and molecular levels compared to control AAV8-treated mice (Supplementary Fig. 8b-e). Moreover, AAV8-hAdi-Cre-treated mice showed increased tolerance to acute cold exposure (Supplementary Fig. 8f). Taken together, our results largely support that TET1 acts as a suppressor of browning in inguinal adipose tissue.

TET1 acts as a repressor of the thermogenic gene program. To investigate the mechanism of how TET1 suppresses the thermogenic gene program, we used RNA-Seq to identify the adipocyte-specific target genes of TET1. In short, we profiled fractionated inguinal adipocytes from WT and $\mathrm{KO}$ mice held at RT and exposed to cold for 4-hr (Fig. 4h-n, Supplementary Fig. $9 \mathrm{a}-\mathrm{c}$ ). We found that 51 genes were significantly upregulated and 73 genes were downregulated in the $\mathrm{KO}$ inguinal adipocytes 
a

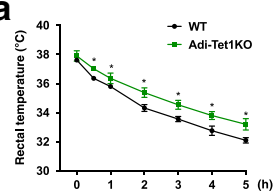

e

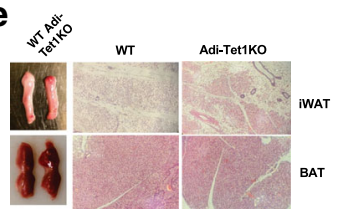

b

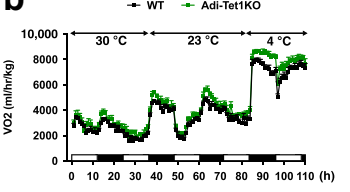

f
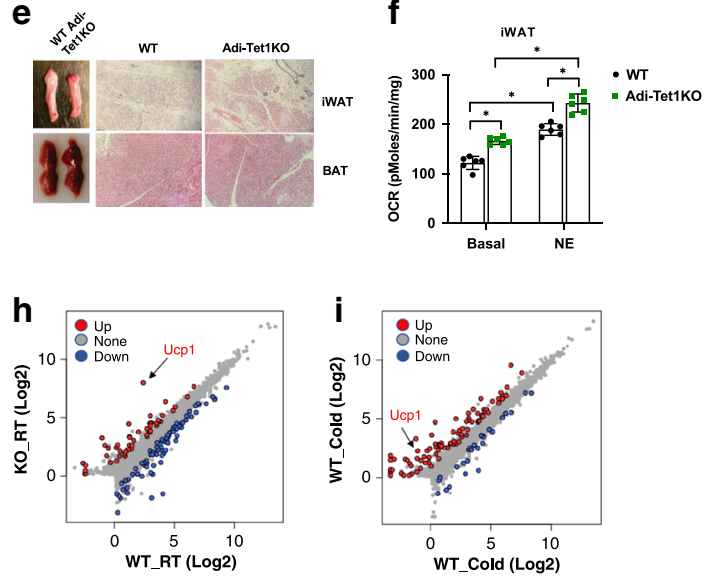

j

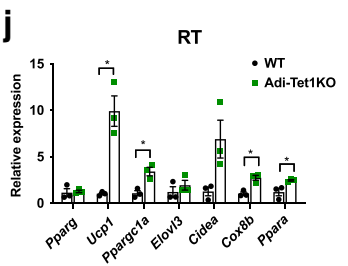

k

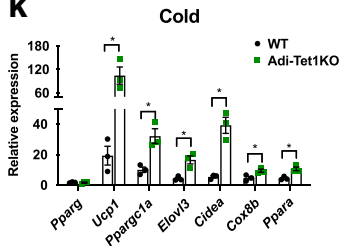

c
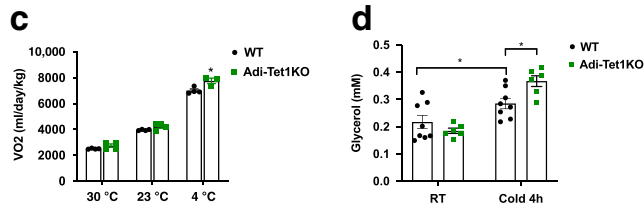

g
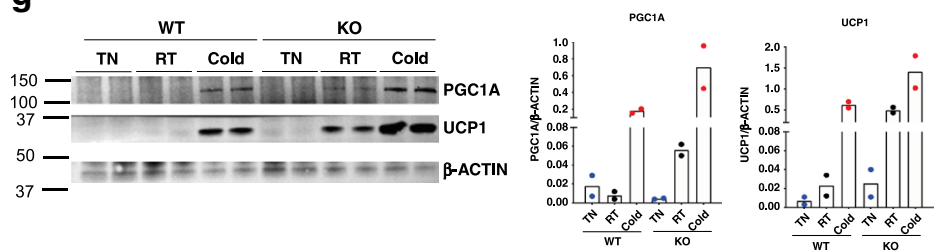

I

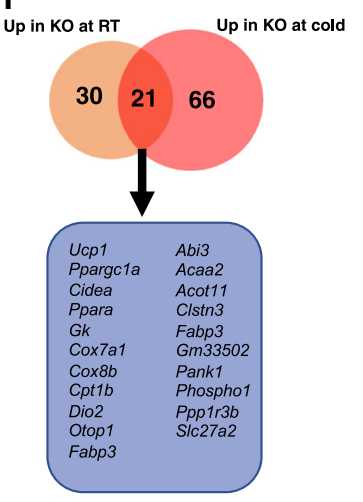

m

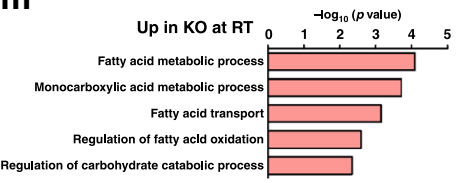

n

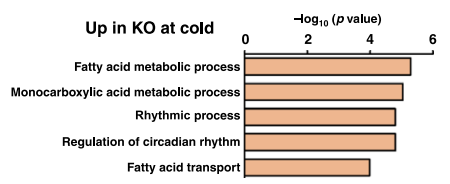

Fig. 4 Adipose-specific Tet1-KO mice show improved cold tolerance. a Rectal core body temperatures of Adi-Tet1-KO and WT mice under cold conditions at indicated time points ( $n=5$ per group. Data are expressed as means \pm SEM. ${ }^{*}$ denotes $p<0.05$, determined by two-tailed Student's $t$ test and one-way ANOVA). b, c Shown is the whole-body oxygen consumption rate $(\mathrm{VO} 2, \mathbf{b})$ and averaged VO2 $\mathbf{c}(n=4$ per group. Data are expressed as means \pm SEM. *denotes $p<0.05$, determined by two-tailed Student's $t$ test and two-way ANOVA followed by Bonferroni post-hoc testing). $\mathbf{d}$ Serum levels of glycerol were measured from WT and KO mice that were housed at RT or exposed to cold for $24 \mathrm{~h}$ ( $n=8 \mathrm{WT}, n=6 \mathrm{KO}$. Data are expressed as means \pm SEM. *denotes $p<0.05$, determined by two-tailed Student's $t$ test and two-way ANOVA followed by Bonferroni post-hoc testing). e Whole tissue and H\&E staining of iWAT and BAT from WT and KO mice that were exposed to cold for 7 days. $\mathbf{f}$ Oxygen consumption rate of iWAT from WT and KO mice was measured with and without stimulation of NE. Average basal respiration rate is presented with or without norepinephrine addition ( $n=6$ per group. Data are expressed as means \pm SEM. ${ }^{\star}$ denotes $p<0.05$, determined by two-tailed Student's $t$ test and two-way ANOVA followed by Bonferroni post-hoc testing). $\mathbf{g}$ Immunoblot of UCP1 and PGC1A from WT and KO iWAT from RT, exposed to TN or cold for 7 days. The relative expression is shown by normalizing to $\beta$-ACTIN ( $n=2$ per group. Data are presented as means of the two). $\mathbf{h}$, i Scatter plot showing differentially regulated genes in inguinal $\mathrm{KO}$ adipocytes from WT and KO mice held at RT and exposed to cold for $4 \mathrm{~h}$ ( $n=2$ per group for RT, $n=3 \mathrm{WT}, n=2 \mathrm{KO}$ for Cold, FC $>1.5, \mathrm{FDR}<0.05$ ). j, $\mathbf{k}$ q-PCR analysis of key thermogenic genes from WT and KO inguinal adipocytes from WT and KO mice at RT and cold exposed for $4 \mathrm{~h}$ ( $n=3$ per group. Data are expressed as means \pm SEM. *denotes $p<0.05$, determined by two-tailed Student's $t$ test). I Venn diagram of the upregulated genes in the KO adipocytes at RT vs cold conditions and the gene names that are commonly upregulated in the KO adipocytes at both temperatures ( $n=2$ per group for RT, $n=3 \mathrm{WT}, n=2 \mathrm{KO}$ for Cold). Gene list is provided in Supplementary Table 1. $\mathbf{m}, \mathbf{n}$ Commonly upregulated biological pathways in the KO adipocytes from RT and cold. Source data are provided as a source data file.

at RT, and 87 genes were upregulated and 26 genes downregulated after a 4 -hr cold exposure (Fig. 4 h, i, l, Supplementary Fig. 9b). This suggests that TET1 acts as both a gene repressor and activator in inguinal adipocytes. Interestingly, the upregulated genes in the $\mathrm{KO}$ adipocytes greatly overlapped between RT and cold conditions (Fig. 4l), and many of those are involved in thermogenesis (e.g., Ucp1, Pgc1a, Cidea, and Elovl3). These were validated by qPCR analysis (Fig. $4 \mathrm{j}, \mathrm{k}$ ). This suggests that the transcriptional regulation of key thermogenic genes in the $\mathrm{KO}$ is already primed under RT, which is mildly cold for mice. By contrast, little overlap was found between the genes that are downregulated in the $\mathrm{KO}$ adipocytes between RT and cold (Supplementary Fig. 9b). Gene Ontology term analysis found that the commonly upregulated pathways in $\mathrm{KO}$ inguinal adipocytes are relevant to thermogenesis, such as fatty acid metabolism, under both RT and cold (Fig. $4 \mathrm{~m}, \mathrm{n}$ ).
Tet1 KO mice are protected against diet-induced obesity. Increased browning leads to increased energy expenditure and improved glucose homeostasis ${ }^{18,19}$. Thus, we assessed whether the increased browning during adipose-selective Tet 1 deficiency leads to metabolic improvement. Despite the body weight of AdiTet1KO remaining unchanged (Fig. 5a), their fat mass was significantly reduced compared to their littermate controls on a chow diet (Fig. 5b). Reduced adiposity in Adi-Tet1KO mice on chow was accompanied by improved glucose tolerance, insulin sensitivity, and hypoinsulinemia (Fig. 5c-e). Next, we asked whether adipose-selective Tet1 deficiency confers protection against diet-induced obesity and impaired glucose tolerance by placing cohort mice on a high-fat diet (HFD, 60\% calories from fat). The body weights of the two groups began to significantly diverge after 6 weeks of HFD feeding (Fig. 5f). The KO had reduced fat mass (Fig. 5g), and their tissue mass of inguinal and 

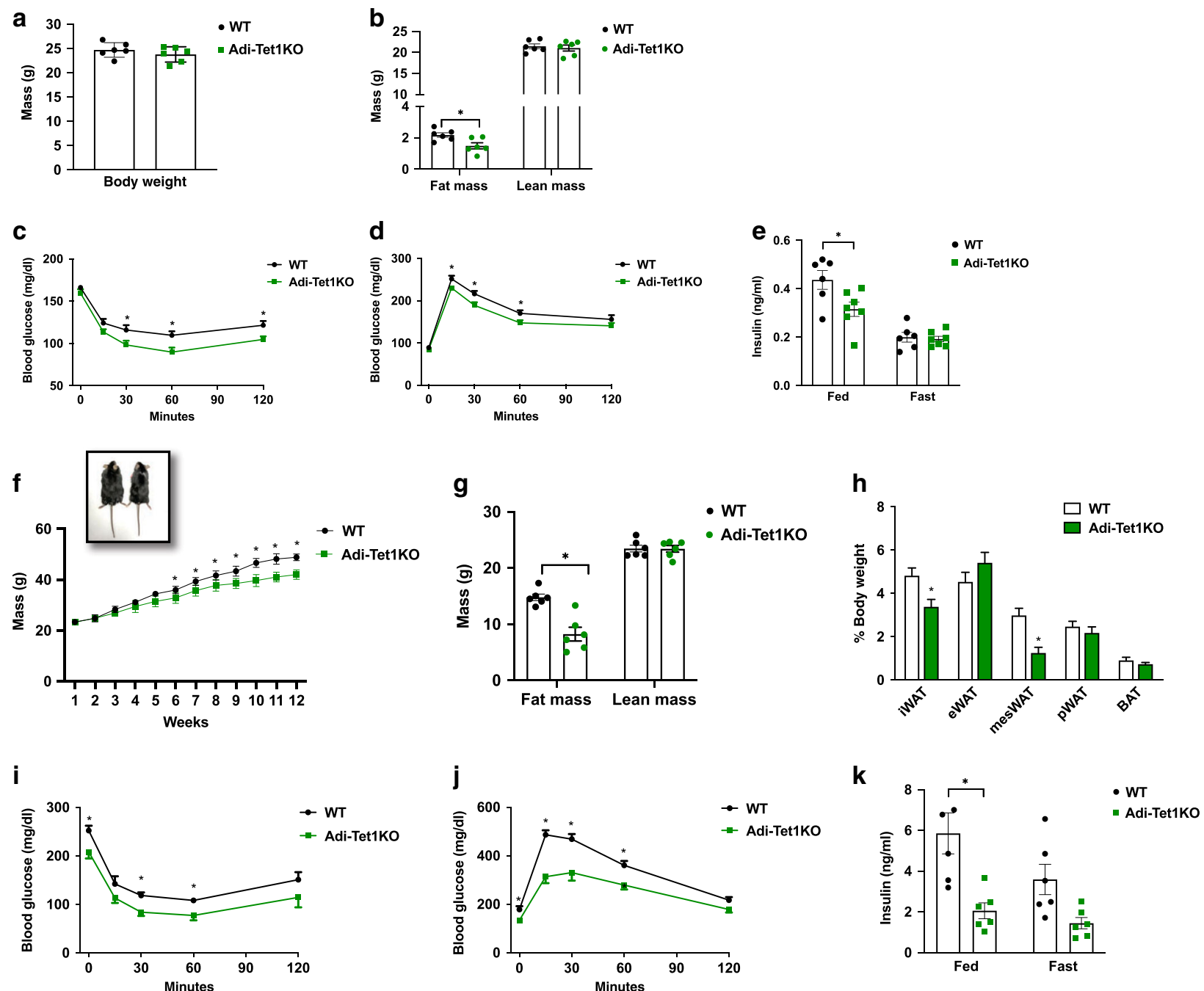

Fig. 5 Adipose-specific Tet1-KO mice are protected from diet-induced obesity and metabolic dysregulation. a, b Body weight (a) and body composition by EchoMRI (b) of 8 -week-old WT and KO mice on a chow diet ( $n=6$ per group. Data are expressed as means \pm SEM. ${ }^{\star}$ denotes $p<0.05$, determined by two-tailed Student's $t$ test). c, d Insulin tolerance test (c) and glucose tolerance test (d) on chow diet $(n=8 \mathrm{WT}, n=6 \mathrm{KO}$. Data are expressed as means \pm SEM. *denotes $p<0.05$, determined by two-tailed Student's $t$ test). e Fed and fasted insulin levels from a chow-fed cohort ( $n=6$ per group. Data are expressed as means \pm SEM. *denotes $p<0.05$, determined by two-tailed Student's $t$ test and two-way ANOVA followed by Bonferroni post-hoc testing). f Weekly body weight of WT and KO mice on a high-fat diet ( $n=7$ per group. Data are expressed as means \pm SEM. ${ }^{*}$ denotes $p<0.05$, determined by twotailed Student's $t$ test). $\mathbf{g}$ Body composition after 10 weeks of HFD ( $n=6$ per group, Data are expressed as means \pm SEM. *denotes $p<0.05$, determined by two-tailed Student's $t$ test). h Adipose tissue weight from HFD cohort ( $n=6$ per group. Data are expressed as means \pm SEM. ${ }^{*}$ denotes $p<0.05$,

determined by two-tailed Student's $t$ test). $\mathbf{i}, \mathbf{j}$ Insulin tolerance test (i) and glucose tolerance test (j) after 10 or 11 weeks on HFD, respectively ( $n=6$ per group, Data are expressed as means \pm SEM. * denotes $p<0.05$, determined by two-tailed Student's $t$ test). $\mathbf{k}$ Fed and fasted insulin levels from high-fat-fed mice ( $n=6$ per group. Data are expressed as means \pm SEM. *denotes $p<0.05$, determined by two-tailed Student's $t$ test and Two-way ANOVA followed by Bonferroni post-hoc testing). Source data are provided as a source data file.

mesenteric WAT was significantly decreased (Fig. 5h). Indirect calorimetry analysis by CLAMS revealed that the KO mice had increased energy expenditure (Supplementary Fig. 10a, b) without changes in food intake and locomotor activity (Supplementary Fig. 10c, d). Interestingly, the KO had an increased RER on HFD (Supplementary Fig. 10e), suggesting that they prefer carbohydrates over fat as a main energy source on HFD. Consistent with the lean phenotype, $\mathrm{KO}$ mice were more glucose tolerant and insulin sensitive than controls, having reduced insulin levels at fed and fast states on HFD (Fig. 5i-k).

TET1 acts in a DNA demethylase-independent manner. TETs mediate their biological functions in both DNA demethylasedependent and -independent manners $27-31$. Thus, we addressed whether demethylation activity is necessary for TET1 to suppress the thermogenic program in beige adipocytes. First, as a genetic approach, we generated various Tet1 mutant alleles (Fig. 6a). Lentiviral overexpression of a Tet1 mutant allele that lacks cterminal catalytic activity $(\text { Tet } 1 \Delta \mathrm{CD})^{42}$ (Fig. 6b) still repressed Ucp1 transcription to a similar degree as the wild-type allele (Tet1 WT) (Fig. 6c). On the other hand, overexpressing either the truncation mutant that contains only the catalytic domain (Tet1 CD) or the catalytically inactive mutant with two critical amino acid substitutions (Tet1 CDM $)^{43}$ was not able to repress Ucp1 expression (Fig. 6c). Both Tet1 WT and Tet1 $\Delta \mathrm{CD}$ equally inhibited mitochondrial respiration in the presence of norepinephrine (Fig. 6d, e).

Second, we performed Tet1 loss-of-function studies in cells that had all three DNA methyltransferases (Dnmts) knocked down to reduce the level of $5 \mathrm{mC}$ (Supplementary Fig. 11a), which is the 
a

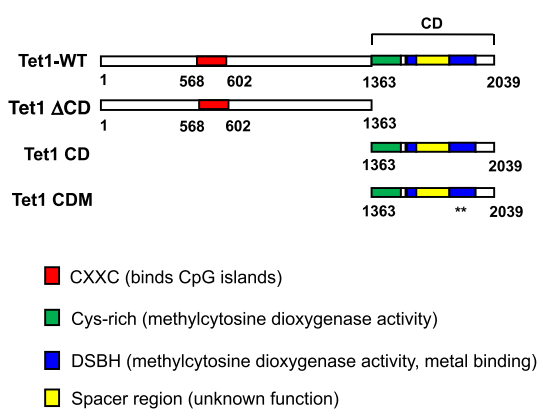

b

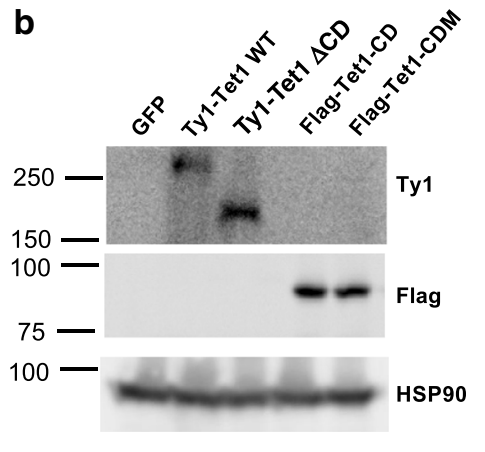

C

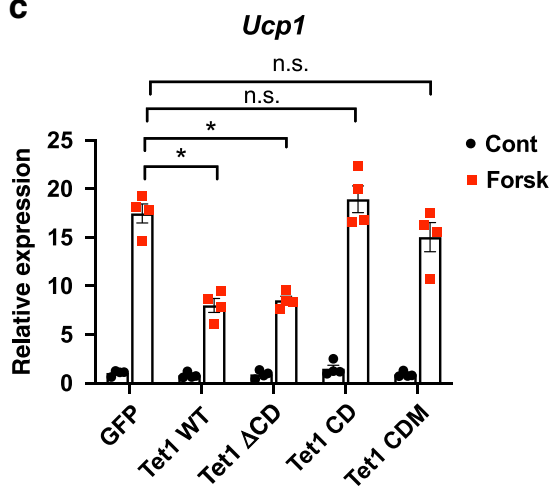

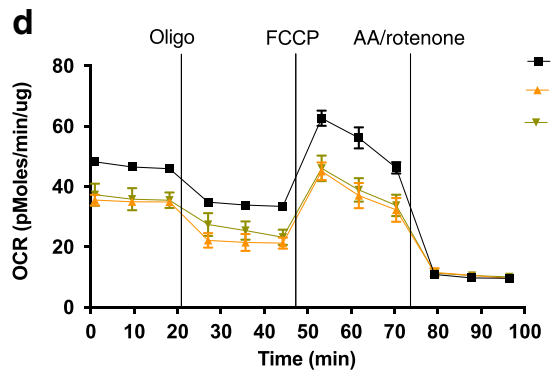

f

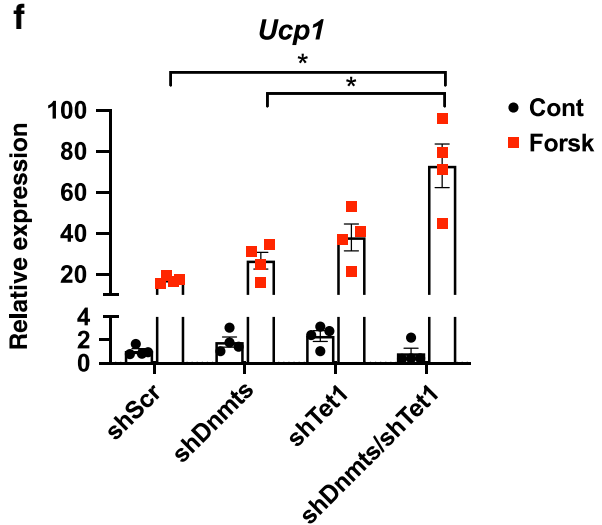

e

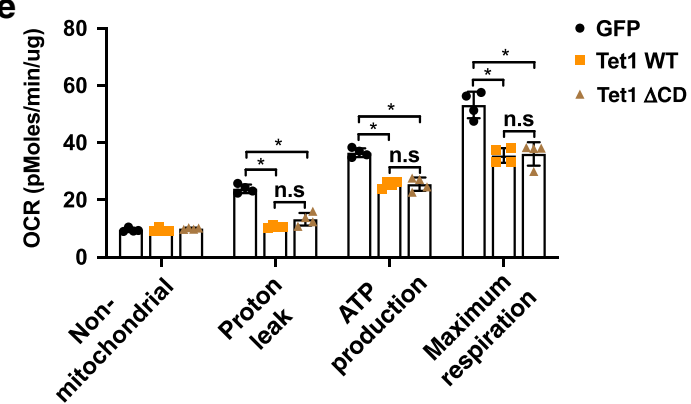

g Ucp1

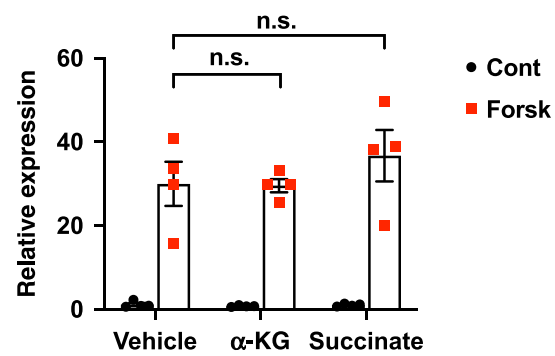

Fig. 6 TET1 suppresses the thermogenic gene program in a DNA demethylase-independent manner. a The protein map showing Tet1 wild-type and mutant alleles: the Tet1 wild-type (Tet1 WT) allele, a truncation mutant that lacks DNA demethylase activity (Tet1 $\triangle$ CD), a mutant that contains only DNA demethylase activity (Tet1 CD), and a catalytically inactive form (Tet1 CDM). b Mature beige adipocytes were transduced with lentiviral expression plasmids for the various Tet1 alleles from (a). c The basal- and forskolin-stimulated levels of Ucp1 were measured by qPCR from (b) ( $n=4$ per group. Data are expressed as means \pm SEM. *denotes $p<0.05$, determined by two-tailed Student's $t$ test and one-way ANOVA followed by Bonferroni post-hoc testing). d, e Mitochondrial respiration is measured by Seahorse from beige adipocytes that overexpress Tet1 WT, Tet1 $\Delta$ CD, or GFP. ( $n=4$ per group. Data are expressed as means \pm SEM. *denotes $p<0.05$, determined by two-tailed Student's $t$ test and two-way ANOVA followed by Bonferroni post-hoc testing). f Beige adipocytes were transduced with hairpins against Dnmt1, 3a, and, $3 b$ or control scramble RNA (shScr), and differentiated beige adipocytes were transduced with shTet1. The basal- and forskolin-stimulated levels of Ucp1 were measured by qPCR ( $n=4$ per group. Data are expressed as means \pm SEM. *denotes $p<0.05$, determined by two-tailed Student's $t$ test and two-way ANOVA followed by Bonferroni post-hoc testing). $\mathbf{g}$ Alpha-KG ( $\alpha$-KG), diethyl-succinate (Succinate), or a vehicle was used to pre-treat mature beige adipocytes for $3 \mathrm{hr}$. The basal- and forskolin-stimulated levels of Ucp1 were measured by qPCR ( $n=4$ per group. Data are expressed as means \pm SEM. *denotes $p<0.05$, determined by two-tailed Student's $t$ test and two-way ANOVA followed by Bonferroni post-hoc testing). Source data are provided as a source data file.

enzymatic substrate for TET. If DNA demethylation activity was critical, the effect of Tet 1 loss-of-function would be diminished in these cells. Interestingly, knocking down the Dnmts alone resulted a trend toward an increase in the Ucp1 expression, and double knockdown with Tet1 further increased the expression of Ucp1 (Fig. 6f). Third, we manipulated TET demethylase activity at the co-factor level. Alpha-ketoglutarate $(\alpha-K G)$, a key metabolite from the TCA cycle, acts as a co-factor for the demethylation activity of TETs, whereas another TCA intermediate, succinate, inhibits TET activity ${ }^{44,45}$. We treated mature brown and beige adipocytes with cell-permeable forms of $\alpha-K G$ and diethylsuccinate then subjected them to forskolin treatment. Neither metabolite had any significant impact on forskolin-stimulated Ucp1 expression (Fig. 6g).

Third, we profiled genome-wide maps of $5 \mathrm{hmC}$, a major intermediate product of TET-mediated oxidation of methylated cytosine $(5 \mathrm{mC})$, using the sensitive $5 \mathrm{hmC}$-Seal-Seq method in iWAT from C57BL/6 J WT mice housed at RT or exposed to cold 
for 7 days (Supplementary Fig. 12). We found no significant changes in $5 \mathrm{hmC}$ levels at the key thermogenic genes under cold conditions, as exemplified at the Ucp1 and Ppargcla loci (Supplementary Fig. 12a, b). This suggests that $5 \mathrm{hmC}$ accumulation is not a key epigenetic event underlying the temperature-triggered browning of beige adipocytes. Taken together, these results suggest that TET1mediated suppression of the thermogenic gene program is largely done in a DNA demethylase-independent manner.

Lastly, we performed bisulfite PCR sequencing to assess the base-pair resolution of DNA methylation changes at several CpGrich Ucp1 and Tet1 promoter/enhancer regions ${ }^{46-49}$. We noted an overall reduction of $\mathrm{CpG}$ methylation at one of the $U c p 1$ promoter regions (named Ucpl (\#1) under lower temperature conditions (Supplementary Fig. 13a, e). Other than that, no obvious changes in average $\mathrm{CpG}$ methylation were detected in the Ucp1 and Tet1 promoter/enhancer regions that we tested (Supplementary Fig. 13b-d, f-h, o-q, r-t). In terms of methylation at individual $\mathrm{CpGs}$, some of the $\mathrm{CpG}$ sites tended to be hypermethylated while some were rather hypomethylated at lower temperatures or showed no particular correlation with temperature conditions (Supplementary Fig. 13i-l, $\mathrm{u}-\mathrm{w}$ ). Future studies are warranted to determine the functional significance of the changes at individual $\mathrm{CpG}$ sites.

TET1 coordinates with HDAC1 to mediate the thermogenic gene repression. It has been proposed that the repressor role of TET1 in gene regulation in other cell types is accomplished through interacting with other repressor proteins like polycomb repressive complex $2(\mathrm{PRC} 2)^{50}, \mathrm{HDACs}^{51}$, and SIN3A ${ }^{52}$. Since genetic and pharmacological inhibition of HDAC1 increases UCP1 and PGCla expression and oxidative metabolism ${ }^{53-55}$. we sought to determine whether TET1-mediated repression involves interacting with HDACl. First, we detected an interaction between TET1 and HDAC1 by co-immunoprecipitating in HEK293T cells (Fig. 7a). Interestingly, the interaction with $\mathrm{HDAC1}$ still existed with Tet1 $\triangle \mathrm{CD}$, which lacks DNA demethylase activity at the cterminal region (Fig. 7a). We examined whether TET1 and $\mathrm{HDAC} 1$ are recruited to the same gene regulatory regions of $U c p 1$ and Ppargcla $a^{55}$. Since we were not able to identify a high-quality antibody against endogenous TET1, we immunoprecipitated TET1 tagged with of Tyl tag from overexpressor cells. With ChIPreChIP analysis, we confirmed the simultaneous binding of HDAC1 and TET1 at these regulatory regions (Fig. $7 \mathrm{~b}$ ) and that both TET1 and HDAC1 binding at these sites was greatly diminished with forskolin stimulation (Fig. 7c-e).

HDAC1 deacetylates histones, which prevents transcription. Therefore, to examine the downstream epigenetic changes that TET1 loss-of-function confers, we performed ChIP-PCR using the active histone mark H3K27ac in WT and KO adipose tissue harvested from different temperature conditions. As expected, in WT iWAT samples, H3K27ac enhancer activity was higher at RT and highest during cold exposure as compared to TN. Overall, this temperature-dependent H3K27ac enhancer activity was even more dramatic in KO iWAT (Fig. 7f-h), in concert with increased expression of Ucp1 and Ppargcla (Fig. 4j, k). Lastly, we assayed the role of HDAC1 in TET1-mediated thermogenic gene suppression. CRISPR-Cas-mediated knockout of HDAC1 (Supplementary Fig. 11b) fully rescued TET1mediated repression of Ucp1 (Fig. 7i, j). Together, our results suggest that the role of TET1 as a suppressor of the thermogenic gene program is in large part due to HDAC1.

\section{Discussion}

While classical brown and beige adipocytes share many fundamental features in morphology and function, discrete characteristics have been identified, including the plasticity of beige thermogenesis ${ }^{1}$. A recent epigenomic profiling study demonstrated that the temperature-mediated plasticity of subcutaneous fat is accompanied by profound changes in chromatin state $^{10}$. To add to these results, we identified TET1 as a beige fatselective epigenetic repressor of the thermogenic gene program.

We demonstrated that TET1 represses thermogenic gene regulation in a largely DNA demethylase-independent manner. In support of this notion, transcriptional changes induced by overexpression of TET1 were highly similar to those induced by its demethylation activity-dead mutant in differentiated cell lines. Other studies have demonstrated that TETs, in addition to modifying cytosine methylation, can act as gene regulators through a DNA methylation-independent manner ${ }^{28}$. The repressive role of TET1 in transcriptional regulation has been proposed to derive from its interaction with other repressor proteins. In ES cells, but not in somatic cells, TET1 contributes to silencing some genes by interacting with polycomb repressive complex 2 (PRC2), which targets repressive histone mark H3K27me350. TET1 is also found in the repressor complex containing SIN3A and HDAC1/2 in both mESCs and HEK293T cells, and its localization greatly overlaps with the SIN3A binding profile genome-wide ${ }^{30}$. Thus, our results are in line with these studies, as TET1 cooperates with HDAC1 to repress some of the key thermogenic genes like Ucp1 and Ppargcla. We speculate that such interaction facilitates local epigenetic modifications and regulates other transcription factors and co-factors to regulate transcriptional activity.

There is also indirect evidence that DNA demethylation and TETs are also required for the development of interscapular $\mathrm{BAT}^{56}$. In this study, the authors suggested that TET proteins mediate demethylation at the promoter region of $\operatorname{Prdm} 16^{56}$, a key developmental gene that is critical for the brown adipogenic lineage and maintains brown adipocyte identity. Moreover, reducing the level of $\alpha$-ketoglutarate ( $\alpha-K G)$, a co-factor for the TET enzymes, leads to reduced demethylation of Prdm16 and impaired brown adipocyte development and function in mutant mice carrying loss-of-function AMPKa $1^{56}$. Given the proadipogenic function of TET1 in white and brown adipogenesis, our results demonstrating TET1 is an inhibitor of thermogenic genes may seem contradictory. However, such dual roles have been reported with other "whitening" transcription factors. For example, Zfp423, preferentially expressed in white adipocytes, is a pro-adipogenic commitment factor in vitro and in vivo ${ }^{57}$, but it also acts as a molecular gate keeper that maintains white cell identity while suppressing browning in mature adipocytes ${ }^{58}$. Interestingly, a recent study reported that the glucocorticoid receptor (GR) drives beige adipocyte whitening as an upstream regulator of Zfp423 ${ }^{10}$. TLE3, another whitening transcription factor, promotes adipose conversion during early differentiation by interacting with PPAR $\gamma$ and antagonizing Wnt signaling 59 . But a recent study identified its novel role as a suppressor of thermogenic gene expression in beige adipocytes ${ }^{60}$. Notably, both Zfp 423 and TLE3 inhibit the activity of pro-browning transcription factor early B-cell factor $2(\mathrm{EBF})^{58,60}$, which cooperates with PPAR $\gamma$ and epigenetic modifiers, such as the chromatin remodelers BRG1 and $\mathrm{BAF}$, and a long noncoding RNA, Blnc1 ${ }^{61}$. Future studies are warranted to determine whether TET1-mediated gene repression converges with these known transcriptional regulators.

Notably, two global studies suggest that differences in the DNA methylation profile is not a major contributor to cell type-specific gene expression in white vs beige or brown adipocytes ${ }^{62,63}$. The first global study employed restriction landmark genomic scanning (RLGS) ${ }^{63}$, the method by which methylation-sensitive restriction enzymes preferentially cut $\mathrm{CpG}$ islands in regulatory regions ${ }^{63}$. In this study, authors also did not find a dramatic 
a

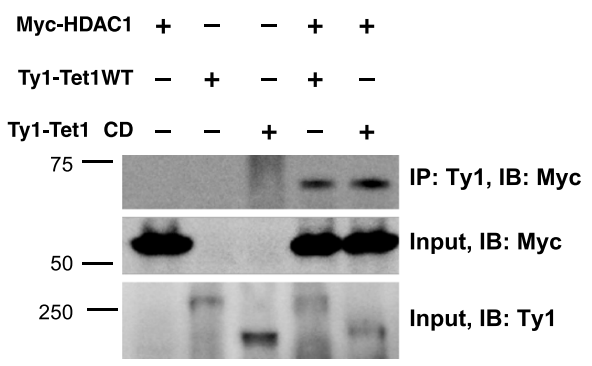

b ChIP-reChIP

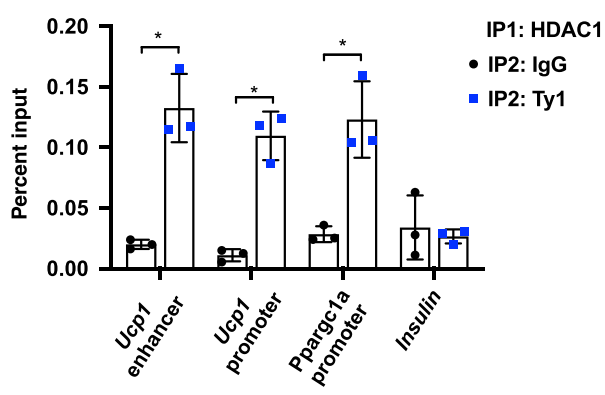

C

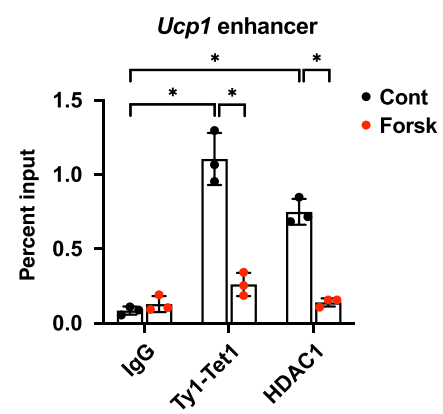

f

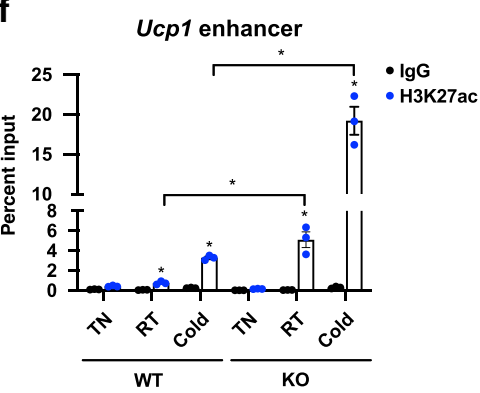

i

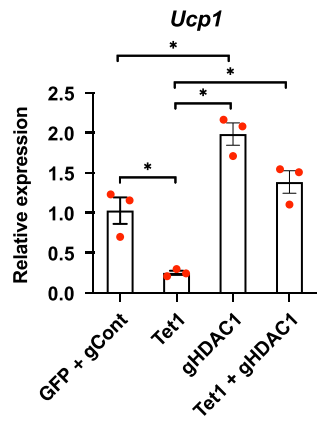

d

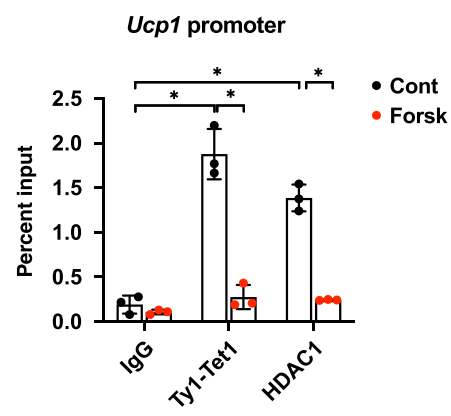

g

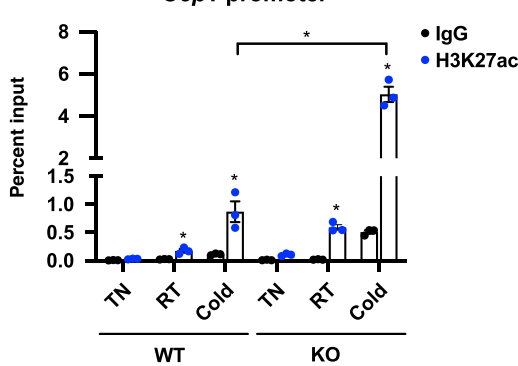

j

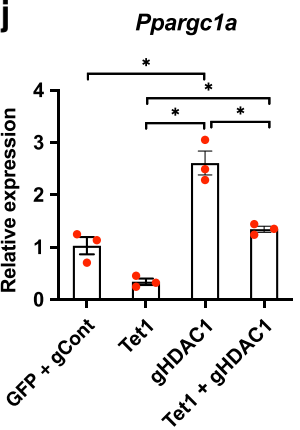

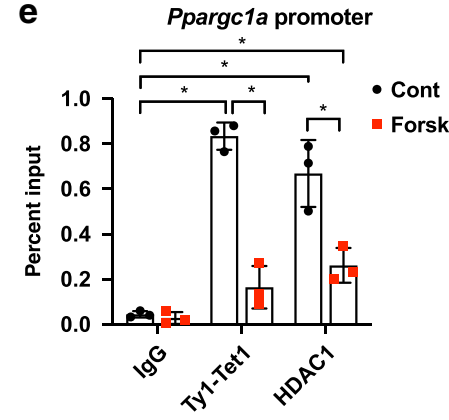

h

h Ppargc1a promoter

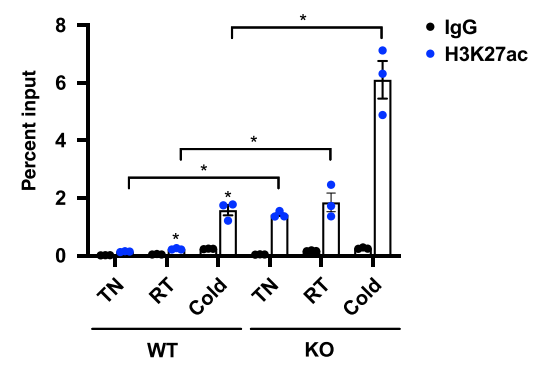

Fig. 7 TET1 coordinates the epigenetic remodeling of the regulatory regions of Ucp1 and Ppargc1a by coordinating with HDAC1. a Co-

immunoprecipitation assay was performed using protein lysates from HEK293T cells that were co-transfected with vectors expressing Ty1-Tet1 WT, Ty1Tet1deltaCD, and Myc-HDAC1. b ChIP-reChIP qPCR analysis was performed on beige adipocytes that were transduced with Ty1-Tet1 WT or GFP using HDAC1 (1st IP), Ty1 (2nd IP), or IgG as a control. The enrichment efficiency is presented as a percent input at the indicated binding sites $(n=3$ per group. Data are expressed as means \pm SEM. *denotes $p<0.05$, determined by two-tailed Student's $t$ test followed by Bonferroni post-hoc testing). c-e Ty1 and HDAC1 ChIP-qPCR analysis was performed in cells that express Ty1-Tet1 WT with and without forskolin stimulation ( $n=3$ per group. Data are expressed as means \pm SEM. *denotes $p<0.05$, determined by two-tailed Student's $t$ test and two-way ANOVA followed by Bonferroni post-hoc testing). f-h H3K27ac ChIP-qPCR analysis was performed in WT and KO iWAT from RT or exposed to TN or cold for 7 days ( $n=3$ per group). Data are expressed as means \pm SEM. *denotes $p<0.05$, determined by two-tailed Student's $t$ test and two-way ANOVA followed by Bonferroni post-hoc testing. $\mathbf{i}, \mathbf{j}$ Beige adipocytes were transduced with either Tet1 overexpressor, gRNA against Hdac1, both, or control plasmids. The forskolin-stimulated level of Ucp1 and Ppargcla was measured by qPCR ( $n=4$ per group, Data are expressed as means \pm SEM. *denotes $p<0.05$, determined by two-tailed Student's $t$ test and two-way ANOVA followed by Bonferroni post-hoc testing). Source data are provided as a source data file. 
difference in the DNA methylation profile between primary white vs. brown adipocytes. The other global study profiled in vitrodifferentiated white and brown adipocytes from inguinal and brown adipose depots using reduced representation bisulfite sequencing (RRBS) ${ }^{62,63}$, which focuses on $\mathrm{CpG}$-rich promoter methylation. Although an overall negative correlation between promoter methylation and gene expression was observed when comparing white and brown adipocytes, dramatic differences in DNA methylation at the thermogenic genes were not reported. Consistent with these reports, we did not detect dramatic changes in DNA demethylation at the key thermogenic genes. However, we observed that some of the $\mathrm{CpG}$ sites displayed simultaneous hyper- or hypo-methylation depending on temperature changes at the Ucp 1 promoter region. Thus, genome-wide methylation profiling studies are warranted to more accurately understand the DNA methylation profile under different temperature conditions. Moreover, functional validation studies will be necessary at the level of individual CpGs.

One of the major caveats of our study is the use of Fabp4-Cre, which is expressed in several other non-adipose tissues ${ }^{35,64}$. Thus, we conducted additional experiments to assess whether there is an additional contribution from non-adipose tissues to the increased thermogenesis in Adi-Tet1 KO mice. First, our in vitro gain- and loss-of-function studies showed that Tet1 knockdown and overexpression have a beige adipocyte-autonomous effect on thermogenic gene regulation and mitochondrial respiration. Second, we showed that inguinal-selective knockdown of Tet1 by AAV8-hAdiCre and noted a similar effect on thermogenesis. Third, to address if there is input from macrophage cells in Adi-Tetl $\mathrm{KO}$ mice, we conducted co-culture experiments using beige adipocytes and primary macrophages isolated from Adi-Tet1 KO vs. WT mice. There were no significant changes in basal and forskolin-induced thermogenic gene expression. Lastly, we report no major phenotypic changes in thermogenesis and energy homeostasis in the PDGFRaTet1 KO generated using PDGFRa-Cre, which is expressed in preadipocytes and a variety of tissues, including the lung, heart, intestine, skin, and cranial facial mesenchyme ${ }^{37-39,65-67}$. We, however, note that it is still possible that there could be an additional contribution on top of that from adipose tissues.

In summary, we identified TET1 as an important epigenetic regulator of the thermogenic gene program in beige adipocytes that coordinates with HDAC1. Adipose-specific TET1 loss-offunction led to increased energy expenditure and protection from diet-induced obesity, insulin resistance, and glucose tolerance.

\section{Methods}

Cell culture. Immortalized beige and brown adipocytes were maintained and differentiated with an adipogenic cocktail ( $0.5 \mathrm{mM}$ IBMX, 5uM Rosiglitazone, 5 $\mathrm{mg} / \mathrm{ml}$ insulin, $1 \mathrm{mM}$ dexamethasone). To generate lentivirus particles, lentiviral constructs were co-transfected with pM2DG- and psPAX-expressing plasmids into $293 \mathrm{~T}$ cells. After $48 \mathrm{~h}$, virus-containing supernatant was collected, filtered through $0.45 \mu \mathrm{m}$ filters, and added to mature day 5 adipocytes for $48 \mathrm{~h}$ along with $8 \mu \mathrm{g} / \mathrm{ml}$ Polybrene. Transduction efficiency was determined by comparing to cells transduced in parallel with a GFP-expressing lentivirus. For the ex vivo system, subcutaneous WAT and iBAT from wild-type C57BL/6 mice was fractionated with digestion buffer $\left(10 \mathrm{mg} / \mathrm{ml}\right.$ collagenase D, 2.4 units of dispase II, $10 \mathrm{mM} \mathrm{CaCl}_{2}$ in PBS). The stromal-vascular fraction (SVF) were isolated by $1.5 \mathrm{u} / \mathrm{ml}$ collagenase and plated in culture and differentiated with adipogenic cocktail. For co-culture experiment, beige adipocytes were cultured in 6-well plates and differentiated at day 8 , and macrophages $\left(2 \times 10^{5}\right.$ cells/well $)$ were plated onto the transwell insert containing a $0.4 \mu \mathrm{m}$ polyethylene terephthalate membrane (Costar, Corning, USA) in serum free medium. After incubation together for $24 \mathrm{~h}$, the transwell was removed and beige adipocytes were harvested for analysis. 3T3-L1 adipocytes were differentiated in 12-well plates and then treated with conditioned medium from LPS-activated macrophages from WT and KO mice. 3T3-L1 adipocytes were cultured for $24 \mathrm{~h}$ before gene expression analysis.
}

Reagents. Insulin, dexamethasone, isobutylmethylxanthine (IBMX), a-KG, CL316, 243, diethyl succinate, and thyroid hormone (T3) were purchased from Sigma. Rosiglitazone was purchased from Cayman. Antibodies were purchased from
GeneTex (Tet1, GTX1242071), Thermo Fisher ( $\beta$-actin, MA5-14739), Cell Signaling (HSP-90, 4874), GenScript (Ty1, A01004), Covance (HA, MMS-101R), Sigma (Flag, F3165), Santa Cruz (Myc, SC-40), Abcam (UCP1, Ab10983; PGC1a Ab54481; H3K27ac: Ab4729), Biolegend (CD45-PerCP/Cy5.5,103131; F4/80-PE/ Cy7, Cat\# 123113; Cd11b-Pacific Blue, 101223; CD301-APC, 145707 and eBioscience (Cd11c-PE,12-0114-81).

Animals. Tet $1^{\mathrm{f} / \mathrm{f}}$ mice were obtained from Dr. Anjana Rao laboratory at UCSD. Mice were maintained under a $12 \mathrm{~h} \mathrm{light} / 12 \mathrm{~h}$ dark cycle at room temperature $\left(23^{\circ} \mathrm{C}\right)$ with free access to food and water. For thermal challenge, mice were placed in a cold chamber $\left(4^{\circ} \mathrm{C}\right)$ for up to 1 week or at thermoneutrality $\left(30^{\circ} \mathrm{C}\right)$. Body temperature was measured using a rectal probe (Physitemp). For high-fat feeding studies, male C57BL/6J mice were put on the diet beginning at 8 weeks of age and continuing for up to three months. Blood and various tissue samples were collected. For the CL316,243 studies, animals were given the drug by IP injection for 5 consecutive days at RT and euthanized for the gene expression analysis. For histology, adipose tissues were fixed with neutral-buffered formalin and embedded in paraffin, and sections were stained with H\&E.

iWAT-specific Tet1 Adi-CRE mice were generated by injecting adenoassociated virus (AAV) expressing Adiponectin CRE (AAV8-hAdp-iCre, Vector Biolabs) or control Null (AAV8-Null) into inguinal WAT level. Briefly, mice were anesthetized and maintained using isoflurane. Area around iWAT was shaved on either side of the mice and cleaned with Betadine (\#19-027132,) and Ethanol. A small incision was made to expose the iWAT pads. AAV was injected into both the iWAT depot at 10 different locations per depot of Tet $1 \mathrm{f} / \mathrm{f}$ adult mice using hamilton microsyringe. Viral titer of $5.0 \times 10^{11}$ genomic copies (GC) per mouse. After injection, the incision was closed using suture (\#101-7137, Henry Schein). Efficacy of viral infection and knockdown was evaluated by quantification of TET1 expression. All animal work was approved by the UC Berkeley ACUC.

Protein analysis. Whole-cell protein lysates were prepared using RIPA lysis buffer ( $1 \%$ Triton X-100, $1 \%$ Sodium deoxycholate, $0.1 \%$ SDS, $0.15 \mathrm{M} \mathrm{NaCl}, 50 \mathrm{mM}$ Tris $(\mathrm{pH} 7.2)$ and protease inhibitor cocktail (Complete Mini-EDTA free, 11836170001 , Roche). Protein was resolved using Tris-glycine gels and transferred to PVDF membrane. After blocking with 5\% nonfat dried milk in TBS-Tween $(0.25 \%)$, the membranes were incubated with the appropriate primary antibodies and loading control. Immunoblots were quantified by the ImageJ program.

Immunoprecipitation. HEK-293T cells were transfected with various DNA constructs using Lipofectamine 3000 (Invitrogen). A day after transfection, cells were lysed with RIPA buffer with protease inhibitor cocktail. $500 \mathrm{mg}$ of protein was incubated with the appropriate antibodies overnight. The next day, protein A/G PLUS-Agarose (SC-2003, Santa Cruz) was added and incubated for $2 \mathrm{~h}$, washed with lysis buffer five times and PBS once. Beads were eluted with non-reducing SDS/PAGE loading buffer and subjected to SDS/PAGE and western blotting.

Dot-blot analysis. Genomic DNA was extracted using the DNeasy Blood \& Tissue kit (Qiagen, 69504) following the manufacturer's protocol. DNA was denatured at $95^{\circ} \mathrm{C}$ for $15 \mathrm{~min}$ in $0.1 \mathrm{M} \mathrm{NaOH}$. It was neutralized with $1 \mathrm{M} \mathrm{NH} 4 \mathrm{OAc}$ on ice and diluted to $300 \mathrm{mg}$ in DNAse free water. $200 \mathrm{ng}$ DNA was applied to a positively charged nylon membrane under vacuum with a Dot Blot Microfiltration Apparatus (Bio-Rad). The membranes were briefly washed in $2 \times \mathrm{SSC}$ buffer $(0.3 \mathrm{M} \mathrm{NaCl}$, $30 \mathrm{mM}$ sodium citrate) for $5 \mathrm{~min}$, then baked at $80^{\circ} \mathrm{C}$ for $5 \mathrm{~min}$, and then crosslinked using a UV Stratalinker 1800 . Membranes were blocked with $5 \%$ nonfat dried milk in TBS-Tween $(0.25 \%)$. The membranes were incubated with the $5 \mathrm{mC}$ primary antibodies. For a loading control, membranes were stained with methylene blue. Immunoblots were quantified by the ImageJ program.

Indirect calorimetry. Metabolic rate was measured by indirect calorimetry in open-circuit Oxymaxchambers, a component of the Comprehensive Lab Animal Monitoring System (CLAMS; Columbus Instruments). Mice were housed individually at various temperatures under a $12 \mathrm{~h}$ light/12 h dark cycle. Food and water were available ad libitum.

Cellular and tissue respiration. For cellular respiration, lentivirally transduced beige adipocytes were plated on XF24 Cell Culture Microplates. For tissue respiration, freshly isolated iWAT and iBAT were rinsed in sterile saline and dissected with a microdisector to $2 \mathrm{~mm}$ per well. Oxygen consumption rate (OCR) was determined using an XF24 Extracellular Flux Analyzer (Seahorse Bioscience). Uncoupled and maximal OCR was determined using oligomycin $(4 \mu \mathrm{M})$ and FCCP $(4 \mu \mathrm{M})$. Antimycin A and rotenone $(2 \mu \mathrm{M}$ each) were used to inhibit Complex IIIand Complex I-dependent respiration.

ChIP-qPCR. Cells were cross-linked with $1 \%$ formaldehyde for $10 \mathrm{~min}$ at room temperature. Cross-linked chromatin was sonicated using an S220 Ultrasonicator (Covaris) to generate DNA fragments of $\sim 200-500 \mathrm{bp}$. Inputs were taken from cleared lysates, and the rest were rotated $\mathrm{O} / \mathrm{N}$ at $4{ }^{\circ} \mathrm{C}$ with $\mathrm{Ty} 1, \mathrm{HDACl}$, and IgG antibodies for immunoprecipitation. An aliquot of $20 \mu \mathrm{l}$ of pre-washed Dynabeads 
Protein $\mathrm{G}$ was added per IP and rotated $1 \mathrm{~h}$ at $4{ }^{\circ} \mathrm{C}$. Beads were successively washed in low-salt RIPA buffer (20 mM Tris-HCl [pH 8.0], 1 mM EDTA, $1 \%$ Triton $\mathrm{x}-100$, $0.1 \%$ SDS, $140 \mathrm{mM} \mathrm{NaCl}, 0.1 \% \mathrm{Na}$ deoxycholate), high-salt RIPA buffer $(20 \mathrm{mM}$ Tris-HCl [pH 8.0], $1 \mathrm{mM}$ EDTA, $1 \%$ Triton $\mathrm{x}-100,0.1 \%$ SDS, $500 \mathrm{mM} \mathrm{NaCl}, 0.1 \%$ $\mathrm{Na}$ deoxycholate), $\mathrm{LiCl}$ buffer (250 mM LiCl, $0.5 \% \mathrm{NP} 40,0.5 \% \mathrm{Na}$ deoxycholate, $1 \mathrm{mM}$ EDTA, $10 \mathrm{mM}$ Tris-HCl [pH 8.0]) and TE buffer (10 mM Tris-HCl [pH 8.0] and $1 \mathrm{mM}$ EDTA). Each reaction was then incubated in digestion buffer $(50 \mathrm{mM}$ Tris- $\mathrm{HCl}$ [pH 8.0], $1 \mathrm{mM}$ EDTA, $100 \mathrm{mM} \mathrm{NaCl}, 0.5 \%$ SDS, proteinase $\mathrm{K}$ ) for a minimum of $4 \mathrm{~h}$ at $65^{\circ} \mathrm{C}$ to reverse cross-links. DNA was recovered using a phenol-chloroform extraction. ChIP-reChIP was performed in essentially the same way as ChIP, except that the first elution was carried out in a digestion buffer (50 mM Tris, pH 8.0, $1 \mathrm{mM} \mathrm{EDTA}, 1 \% \mathrm{SDS}, 50 \mathrm{mM} \mathrm{NaHCO}_{3}$ ) at $65^{\circ} \mathrm{C}$ for $10 \mathrm{~min}$. After saving the supernatant, the beads were with $40 \mathrm{ul} 10 \mathrm{mM}$ DTT for $30 \mathrm{~min}$ at $37^{\circ} \mathrm{C}$. The combined elutes were subjected to the second IP. Real-time qPCR primers are listed in Supplementary Table 2. All data were normalized to input.

RNA extraction and quantitative PCR. Total RNA was extracted from cells or tissues using TRIzol reagent according to the manufacturer's instructions. cDNA was reverse-transcribed from $1 \mu \mathrm{g}$ of RNA using the RETROscript first strand synthesis kit (Ambion). Quantitative PCR (qPCR) was performed with SYBR Green qPCR Master Mix (Applied Biosystems) using a 7900HT Fast Real-Time PCR System (Applied Biosystems) and CFX96 Touch (BioRad). Primer sequences are listed in Supplemental Table 2. The relative amount of mRNA normalized to cyclophilin B was calculated using the delta-delta method ${ }^{68}$.

RNA-Seq analysis. RNA samples were extracted using the RNeasy Mini kit (Qiagen, 74104) following the manufacturer's protocol. Libraries were prepared using the BGI Library Preparation Kit, and sequencing was performed on the BGISEQ. RNA-Seq reads were aligned to UCSC mm10 genome using STAR aligner ${ }^{69}$ with an option, “--outFilterMultimapNmax 1". Mitochondrial reads were filtered out to avoid sequencing depth bias due to mitochondrial abundance. Then, raw read count for each gene was measured using Feature Counts. Differential gene expression analysis was performed using edgeR. Hierarchical clustering was performed using group-wise average gene expression levels to identify distinct functional modules of genes using Ward's criterion and Pearson correlation as a similarity measure. Gene ontology analysis was done using EnrichR.

5hmC-seal analysis. Genomic DNA (250 ng) was sonicated to $~ 100-500$ bp with a Bioruptor PICO sonicator (Diagenode). Sonicated DNA was end-repaired, Atailed, and ligated to paired-end adapters following the standard Illumina protocol. The glucosylation reactions were performed in a $50 \mu$ l solution containing $1 \mathrm{x}$ glucosylation buffer, above adapter-ligated DNA, $200 \mu \mathrm{M}$ UDP-Azide-Glucose (Active Motif, 55020), and 5 U T4 ß-glucosyltransferase (Thermofisher, EO0831), at $37^{\circ} \mathrm{C}$ for $1 \mathrm{~h}$. After glucosylation, the reaction was purified by Zymo DNA clean \& concentrator Kit (Zymo, D4014) and eluted into 45ul ddH2O. Then, $1.5 \mu \mathrm{l}$ DBCO-PEG4-Biotin (Click Chemistry Tools, A105. $4.5 \mathrm{mM}$ stored in DMSO, dilute from $30 \mathrm{mM}$ stock before use) was added to the $45 \mathrm{ul}$ glusosylated DNA and the reactions were incubated at $37^{\circ} \mathrm{C}$ for $2 \mathrm{~h}$. Next, the DNA was purified by Zymo DNA clean \& concentrator Kit and eluted in 10ul ddH2O. The purified DNA was pulled down by $5 \mu \mathrm{l}$ streptavidin $\mathrm{C} 1$ beads (Thermofisher, 65001) for $15 \mathrm{~min}$ according to the manufacture's instruction. The beads were subsequently undergone ten washes with $1 \mathrm{x}$ binding-washing buffer and two washes with ddH2O and were resuspended in $15 \mathrm{ul}$ ddH2O. All binding and washing were done at room temperature. The captured DNA fragments were amplified with 12 cycles of PCR amplification using the Phusion DNA polymerase. The PCR products were purified using 1.0X AMPure XP beads according to the manufacture's instruction. DNA concentration of each library was measured with a Qubit fluorometer (Life Technologies) and sequencing was performed on the Next-Seq instrument (Illumina). 5hmC-seq analysis: Sequencing reads was trimmed adapter using cutadapt (https://cutadapt.readthedocs.io/en/stable/), aligned to mouse genome mm10 using BWA with default parameters http://bio-bwa.sourceforge.net/. MethylQA was used to process the aligned BAM files. Concordantly aligned read-pairs were selected and deduplicated using Picard tool (https://broadinstitute.github.io/picard/). Genome browser tracks were created in bigwig files using "makeUCSCfile" in Homer and bedGraphToBigWig in UCSC toolkit.

Bisulfite sequencing. Bisulfite modification was carried out with $250 \mathrm{ng}$ genomic DNA using the EZ DNA Methylation-Lightning Kit (\#D5030 Zymo Research, Irvine, CA) according to the manufacturer's instructions. Promoter and enhancer regions within the Ucp1 and Tet1 genes were amplified using bisulfite PCR. Bisulfite PCR primers were designed with the BiSearch and Methfinder programs, which are listed in Supplementary Table 2. The purified PCR products were cloned into the Topo TA Cloning System (Invitrogen, Carlsbad, CA, USA). Colonies were screened and a minimum of ten clones from each single bisulfite-treated DNA were sequenced, and the data were analyzed for statistical significance.
FACS analysis. SVF cells were incubated with indicated antibody for $20 \mathrm{~min}$ in dark, washed, span at $300 \mathrm{~g}$ for $5 \mathrm{~min}$, resuspended in FACS buffer ( $1 \times$ PBS containing $0.5 \%$ BSA) and passed through $40 \mu \mathrm{m}$ filter prior to FACS analysis. FACS was performed on BD Influx cell sorter. Cells were initially chosen based on forward and side scatter (FFS and CCS) and trigger pulse width. Cells that were not incubated with antibody were used as a control to determine background fluorescence levels.

Plasmids. Hairpins against Dnmt1, Dnmt3a, and Dnmt3b are from Sigma. HDAC1 was subcloned to pcDNA3.1 at EcoRI/NotI for Myc-HDAC1. Lentiviral overexpression vectors for Tet1WT, Tet $1 \Delta \mathrm{CD}$, Tet1CD, and Tet1CDM were subcloned into pCDH using various multicloning sites (XbaI/NotI for TylTet1WT, Ty1-Tet1 $\Delta$ CD, XbaI/NheI for Flag-Tet1CD, Flag-Tet1CDM). Hairpins targeting Tet 1 were subcloned at AgeI/EcoRI or purchased from Open Biosystems Hairpin sequences are shown in Supplemental Table 1. sgRNAs that targeted Hcacl were cloned into lentiCRISPR v2 vector. Hairpin and sgRNA sequences are shown in Supplementary Table 1.

Statistical analyses. Data are presented as means \pm SEM and individual data points are plotted. Sample size was determined by our experience with inherent variability. No statistical method was used to predetermine sample size. Statistical analyses and the number of samples $(n)$ were described in detail for each figure panel. Statistical analyses and the number of samples $(n)$ is described in detail for each figure panel. Two-tailed unpaired Student's $t$ test was used for the comparison between two groups. One-way analysis of variance (ANOVA) or two-way ANOVA followed by the Bonferroni's test was used for the multiple comparisons. Statistical analyses were performed using excel and GraphPad Prism. All reported $p$ values were two-sided and differences were considered significant at $p<0.05$.

Reporting summary. Further information on research design is available in the Nature Research Reporting Summary linked to this article.

\section{Data availability}

The authors declare that the data supporting the findings of this study are available within the paper and its supplementary information files. Global profiling data are available in the GEO repository under accession number: GSE153093. Source data are provided with this paper.

Received: 25 September 2019; Accepted: 27 July 2020; Published online: 27 August 2020

\section{References}

1. Ikeda, K., Maretich, P. \& Kajimura, S. The common and distinct features of brown and beige adipocytes. Trends Endocrinol. Metab. 29, 191-200 (2018).

2. Kajimura, S., Spiegelman, B. M. \& Seale, P. Brown and beige fat: physiological roles beyond heat generation. Cell Metab. 22, 546-559 (2015).

3. Sanchez-Gurmaches, J. et al. PTEN loss in the Myf5 lineage redistributes body fat and reveals subsets of white adipocytes that arise from Myf5 precursors. Cell Metab. 16, 348-362 (2012).

4. Seale, P. et al. PRDM16 controls a brown fat/skeletal muscle switch. Nature 454, 961-967 (2008).

5. Chu, D.-T. \& Gawronska-Kozak, B. Brown and brite adipocytes: same function, but different origin and response. Biochimie 138, 102-105 (2017).

6. Chu, D.-T. \& Tao, Y. Human thermogenic adipocytes: a reflection on types of adipocyte, developmental origin, and potential application. J. Physiol. Biochem. 73, 1-4 (2017)

7. Chu, D.-T., Tao, Y. \& Taskén, K. OPA1 in lipid metabolism: function of OPA1 in lipolysis and thermogenesis of adipocytes. Horm. Metab. Res. 49, 276-285 (2017).

8. $\mathrm{Wu}$, J. et al. Beige adipocytes are a distinct type of thermogenic fat cell in mouse and human. Cell 150, 366-376 (2012).

9. Sharp, L. Z. et al. Human BAT possesses molecular signatures that resemble beige/brite cells. PLoS ONE 7, e49452 (2012).

10. Roh, H. C. et al. Warming induces significant reprogramming of beige, but not brown, adipocyte cellular identity. Cell Metab. 27, 1121-1137.e5 (2018).

11. Grujic, D. et al. $\beta 3$-Adrenergic receptors on white and brown adipocytes mediate $\beta 3$-selective agonist-induced effects on energy expenditure, insulin secretion, and food intake. J. Biol. Chem. 272, 17686-17693 (1997).

12. Ahmadian, M. et al. Desnutrin/ATGL is regulated by AMPK and is required for a brown adipose phenotype. Cell Metab. 13, 739-748 (2011).

13. Altshuler-Keylin, S. et al. Beige adipocyte maintenance is regulated by autophagy-induced mitochondrial clearance. Cell Metab. 24, 402-419 (2016) 
14. Nedergaard, J. \& Cannon, B. UCP1 mRNA does not produce heat. Biochim. Biophys. Acta 1831, 943-949 (2013).

15. Kazak, L. et al. A creatine-driven substrate cycle enhances energy expenditure and thermogenesis in beige fat. Cell 163, 643-655 (2015).

16. Ikeda, K. et al. UCP1-independent signaling involving SERCA2b-mediated calcium cycling regulates beige fat thermogenesis and systemic glucose homeostasis. Nat. Med. 23, 1454-1465 (2017).

17. Cypess, A. M. et al. Identification and importance of brown adipose tissue in adult humans. N. Engl. J. Med. 360, 1509-1517 (2009).

18. Saito, M. et al. High incidence of metabolically active brown adipose tissue in healthy adult humans: effects of cold exposure and adiposity. Diabetes $\mathbf{5 8}$, 1526-1531 (2009).

19. Hanssen, M. J. W. et al. Short-term cold acclimation improves insulin sensitivity in patients with type 2 diabetes mellitus. Nat. Med. 21, 863-865 (2015).

20. Emmett, M. J. et al. Histone deacetylase 3 prepares brown adipose tissue for acute thermogenic challenge. Nature 546, 544-548 (2017).

21. Inagaki, T., Sakai, J. \& Kajimura, S. Transcriptional and epigenetic control of brown and beige adipose cell fate and function. Nat. Rev. Mol. Cell Biol. 17, 480-495 (2016).

22. Ohno, H., Shinoda, K., Ohyama, K., Sharp, L. Z. \& Kajimura, S. EHMT1 controls brown adipose cell fate and thermogenesis through the PRDM16 complex. Nature 504, 163-167 (2013).

23. Sambeat, A. et al. LSD1 Interacts with Zfp516 to Promote UCP1 Transcription and Brown Fat Program. Cell Rep. 15, 2536-2549 (2016).

24. Tateishi, K., Okada, Y., Kallin, E. M. \& Zhang, Y. Role of Jhdm2a in regulating metabolic gene expression and obesity resistance. Nature 458, 757-761 (2009).

25. Zeng, X. et al. Lysine-specific demethylase 1 promotes brown adipose tissue thermogenesis via repressing glucocorticoid activation. Genes Dev. 30, 1822-1836 (2016).

26. Pastor, W. A., Aravind, L. \& Rao, A. TETonic shift: biological roles of TET proteins in DNA demethylation and transcription. Nat. Rev. Mol. Cell Biol. 14, 341-356 (2013).

27. Chen, Q., Chen, Y., Bian, C., Fujiki, R. \& Yu, X. TET2 promotes histone OGlcNAcylation during gene transcription. Nature 493, 561-564 (2013).

28. Gao, J. et al. Non-catalytic roles for TET1 protein negatively regulating neuronal differentiation through srGAP3 in neuroblastoma cells. Protein Cell 7, 351-361 (2016).

29. Tsai, Y.-P. et al. TET1 regulates hypoxia-induced epithelial-mesenchymal transition by acting as a co-activator. Genome Biol. 15, 513 (2014).

30. Williams, K. et al. TET1 and hydroxymethylcytosine in transcription and DNA methylation fidelity. Nature 473, 343-348 (2011)

31. Zhang, Q. et al. Tet2 is required to resolve inflammation by recruiting Hdac2 to specifically repress IL-6. Nature 525, 389-393 (2015).

32. Yoo, Y. et al. TET-mediated hydroxymethylcytosine at the Ppary locus is required for initiation of adipogenic differentiation. Int J. Obes. (Lond.) 41, 652-659 (2017).

33. Eguchi, J. et al. Transcriptional control of adipose lipid handling by IRF4. Cell Metab. 13, 249-259 (2011)

34. He, W. et al. Adipose-specific peroxisome proliferator-activated receptor gamma knockout causes insulin resistance in fat and liver but not in muscle. Proc. Natl Acad. Sci. USA 100, 15712-15717 (2003).

35. Jeffery, E. et al. Characterization of Cre recombinase models for the study of adipose tissue. Adipocyte 3, 206-211 (2014).

36. Nguyen, K. D. et al. Alternatively activated macrophages produce catecholamines to sustain adaptive thermogenesis. Nature 480, 104-108 (2011).

37. Boström, H. et al. PDGF-A signaling is a critical event in lung alveolar myofibroblast development and alveogenesis. Cell 85, 863-873 (1996).

38. Chong, J. J. H. et al. Progenitor cells identified by PDGFR-alpha expression in the developing and diseased human heart. Stem Cells Dev. 22, 1932-1943 (2013).

39. Karlsson, L., Bondjers, C. \& Betsholtz, C. Roles for PDGF-A and sonic hedgehog in development of mesenchymal components of the hair follicle. Development 126, 2611-2621 (1999).

40. O'Neill, S. M. et al. Targeting adipose tissue via systemic gene therapy. Gene Ther. 21, 653-661 (2014).

41. Chella Krishnan, K. et al. Sex-specific metabolic functions of adipose Lipocalin-2. Mol. Metab. 30, 30-47 (2019).

42. Zhang, W. et al. Isoform switch of TET1 regulates DNA demethylation and mouse development. Mol. Cell 64, 1062-1073 (2016).

43. Ito, S. et al. Tet proteins can convert 5-methylcytosine to 5-formylcytosine and 5-carboxylcytosine. Science 333, 1300-1303 (2011).

44. Tahiliani, M. et al. Conversion of 5-methylcytosine to 5hydroxymethylcytosine in mammalian DNA by MLL partner TET1. Science 324, 930-935 (2009).

45. Yang, H. et al. TET-catalyzed 5-methylcytosine hydroxylation is dynamically regulated by metabolites. Cell Res. 24, 1017-1020 (2014).
46. Huang, Z., Bassil, C. F. \& Murphy, S. K. Bisulfite sequencing of cloned alleles. Methods Mol. Biol. 1049, 83-94 (2013).

47. Zhang, Y. et al. DNA methylation analysis by bisulfite conversion, cloning, and sequencing of individual clones. Methods Mol. Biol. 507, 177-187 (2009).

48. Li, L.-C. \& Dahiya, R. MethPrimer: designing primers for methylation PCRs. Bioinformatics 18, 1427-1431 (2002).

49. Itzhak, Y., Ergui, I. \& Young, J. I. Long-term parental methamphetamine exposure of mice influences behavior and hippocampal DNA methylation of the offspring. Mol. Psychiatry 20, 232-239 (2015).

50. Neri, F. et al. Genome-wide analysis identifies a functional association of Tet1 and Polycomb repressive complex 2 in mouse embryonic stem cells. Genome Biol. 14, R91 (2013).

51. Wei, T. et al. An HDAC2-TET1 switch at distinct chromatin regions significantly promotes the maturation of pre-iPS to iPS cells. Nucleic Acids Res. 43, 5409-5422 (2015).

52. Chandru, A., Bate, N., Vuister, G. W. \& Cowley, S. M. Sin3A recruits Tet1 to the PAH1 domain via a highly conserved Sin3-interaction domain. Sci. Rep. 8, 14689 (2018).

53. Galmozzi, A. et al. Inhibition of class I histone deacetylases unveils a mitochondrial signature and enhances oxidative metabolism in skeletal muscle and adipose tissue. Diabetes 62, 732-742 (2013).

54. Ye, J. Improving insulin sensitivity with HDAC inhibitor. Diabetes 62, 685-687 (2013).

55. Li, F. et al. Histone deacetylase 1 (HDAC1) negatively regulates thermogenic program in brown adipocytes via coordinated regulation of histone $\mathrm{H} 3$ lysine 27 (H3K27) deacetylation and methylation. J. Biol. Chem. 291, 4523-4536 (2016).

56. Yang, Q. et al. AMPK/a-Ketoglutarate Axis Dynamically Mediates DNA Demethylation in the Prdm16 Promoter and Brown Adipogenesis. Cell Metab. 24, 542-554 (2016).

57. Gupta, R. K. et al. Transcriptional control of preadipocyte determination by Zfp423. Nature 464, 619-623 (2010).

58. Shao, M. et al. Zfp423 maintains white adipocyte identity through suppression of the beige cell thermogenic gene program. Cell Metab. 23, 1167-1184 (2016).

59. Villanueva, C. J. et al. TLE3 is a dual-function transcriptional coregulator of adipogenesis. Cell Metab. 13, 413-427 (2011).

60. Pearson, S. et al. Loss of TLE3 promotes the mitochondrial program in beige adipocytes and improves glucose metabolism. Genes Dev. 33, 747-762 (2019).

61. Shapira, S. N. et al. EBF2 transcriptionally regulates brown adipogenesis via the histone reader DPF3 and the BAF chromatin remodeling complex. Genes Dev. 31, 660-673 (2017).

62. Lim, Y. C. et al. Dynamic DNA methylation landscape defines brown and white cell specificity during adipogenesis. Mol. Metab. 5, 1033-1041 (2016).

63. Sakamoto, H. et al. Cell type-specific methylation profiles occurring disproportionately in CpG-less regions that delineate developmental similarity. Genes Cells 12, 1123-1132 (2007).

64. Mullican, S. E. et al. A novel adipose-specific gene deletion model demonstrates potential pitfalls of existing methods. Mol. Endocrinol. 27, 127-134 (2013).

65. Karlsson, L., Lindahl, P., Heath, J. K. \& Betsholtz, C. Abnormal gastrointestinal development in PDGF-A and PDGFR-(alpha) deficient mice implicates a novel mesenchymal structure with putative instructive properties in villus morphogenesis. Development 127, 3457-3466 (2000)

66. Lindahl, P. et al. Alveogenesis failure in PDGF-A-deficient mice is coupled to lack of distal spreading of alveolar smooth muscle cell progenitors during lung development. Development 124, 3943-3953 (1997).

67. McCarthy, N. et al. Pdgfra and Pdgfrb genetically interact during craniofacial development. Dev. Dyn. 245, 641-652 (2016).

68. Ljvak, K. J. Analysis of relative gene expression data using real time quantitative PCR and the $2^{\wedge}<-\Delta \Delta C T>$ method. Methods 25, 402-408 (2001).

69. Dobin, A. et al. STAR: ultrafast universal RNA-seq aligner. Bioinformatics 29 , 15-21 (2013)

\section{Acknowledgements}

Work was funded by AHA Award \# 19POST34380834 to D.Y. and R01 DK116008 to S.K. We thank Drs. Hei Sook Sul and Jen-Chywan Wally Wang (UC Berkeley) for helpful conversations about manuscript and thank Dr. Shingo Kajimura (UCSF) for allowing us to learn adenoviral injection to inguinal fat pad in his laboratory.

\section{Author contributions}

S.K. supervised experiments and wrote the manuscript. Experiments were carried out by S.D.V., D.Y., J.K., H.X., P.A., and S.K. H.L. analyzed RNA-Seq and 5hmC-Seal analysis. P.H.J. helped with CLAMS studies. Y.O. helped with adenovirus injections.

\section{Competing interests}

The authors declare no competing interests. 


\section{Additional information}

Supplementary information is available for this paper at https://doi.org/10.1038/s41467020-18054-y.

Correspondence and requests for materials should be addressed to S.K.

Peer review information Nature Communications thanks the anonymous reviewer(s) for their contribution to the peer review of this work. Peer reviewer reports are available.

Reprints and permission information is available at http://www.nature.com/reprints

Publisher's note Springer Nature remains neutral with regard to jurisdictional claims in published maps and institutional affiliations. (c) (i) Open Access This article is licensed under a Creative Commons Attribution 4.0 International License, which permits use, sharing, adaptation, distribution and reproduction in any medium or format, as long as you give appropriate credit to the original author(s) and the source, provide a link to the Creative Commons license, and indicate if changes were made. The images or other third party material in this article are included in the article's Creative Commons license, unless indicated otherwise in a credit line to the material. If material is not included in the article's Creative Commons license and your intended use is not permitted by statutory regulation or exceeds the permitted use, you will need to obtain permission directly from the copyright holder. To view a copy of this license, visit http://creativecommons.org/ licenses/by/4.0/

(C) The Author(s) 2020 